\title{
S100B as a Potential Biomarker and Therapeutic Target in Multiple Sclerosis
}

\author{
Andreia Barateiro ${ }^{1}$ - Vera Afonso ${ }^{1}$ - Gisela Santos ${ }^{1}$ - João José Cerqueira ${ }^{2,3}$. \\ Dora Brites $^{1,4}$ • Jack van Horssen ${ }^{5}$. Adelaide Fernandes ${ }^{1,4}$
}

Received: 19 February 2015 / Accepted: 1 July 2015

(C) Springer Science+Business Media New York 2015

\begin{abstract}
Multiple sclerosis (MS) pathology is characterized by neuroinflammation and demyelination. Recently, the inflammatory molecule S100B was identified in cerebrospinal fluid (CSF) and serum of MS patients. Although seen as an astrogliosis marker, lower/physiological levels of S100B are involved in oligodendrocyte differentiation/maturation. Nevertheless, increased S100B levels released upon injury may induce glial reactivity and oligodendrocyte demise, exacerbating tissue damage during an MS episode or delaying the following remyelination. Here, we aimed to unravel the functional role of S100B in the pathogenesis of MS. Elevated S100B levels were detected in the CSF of relapsing-remitting MS patients at diagnosis. Active demyelinating MS lesions showed increased expression of S100B and its receptor, the
\end{abstract}

Electronic supplementary material The online version of this article (doi:10.1007/s12035-015-9336-6) contains supplementary material, which is available to authorized users.

Adelaide Fernandes

amaf@ff.ulisboa.pt

1 Research Institute for Medicines (iMed.ULisboa), Faculty of Pharmacy, Universidade de Lisboa, Av. Professor Gama Pinto, 1649-003 Lisbon, Portugal

2 Life and Health Sciences Research Institute (ICVS), School of Health Sciences, University of Minho, Campus de Gualtar, 4710-057 Braga, Portugal

3 ICVS/3B's - PT Government Associate Laboratory, Braga Guimarães, Portugal

4 Department of Biochemistry and Human Biology, Faculty of Pharmacy, Universidade de Lisboa, Av. Professor Gama Pinto, 1649-003 Lisbon, Portugal

5 Department of Molecular Cell Biology and Immunology, Neuroscience Campus Amsterdam, VU University Medical Center Amsterdam, P.O. Box 7057, 1007 MB Amsterdam, The Netherlands receptor for advanced glycation end products (RAGE), in the lesion area, while chronic active lesions displayed increased $\mathrm{S} 100 \mathrm{~B}$ in demyelinated areas with lower expression of RAGE in the rim. Interestingly, reactive astrocytes were identified as the predominant cellular source of S100B, whereas RAGE was expressed by activated microglia/macrophages. Using an ex vivo demyelinating model, cerebral organotypic slice cultures treated with lysophosphatidylcholine (LPC), we observed a marked elevation of S100B upon demyelination, which co-localized mostly with astrocytes. Inhibition of S100B action using a directed antibody reduced LPC-induced demyelination, prevented astrocyte reactivity and abrogated the expression of inflammatory and inflammasome-related molecules. Overall, high S100B expression in MS patient samples suggests its usefulness as a diagnostic biomarker for MS, while the beneficial outcome of its inhibition in our demyelinating model indicates S100B as an emerging therapeutic target in MS.

Keywords Cerebellar organotypic slice cultures · Demyelination · Glial inflammatory response $\cdot$ Human samples $\cdot$ Multiple sclerosis $\cdot$ S100B

\section{Introduction}

Multiple sclerosis (MS) is a primary inflammatory demyelinating autoimmune disorder of the central nervous system (CNS) affecting mainly young people aged between 20 and 40 years at disease onset. In early stages of the disease, it is characterized by infiltration and activation of $\mathrm{T}$ cells and accumulation of monocyte-derived macrophages, which promote destruction of the myelin sheath leading to the formation of focal demyelinated lesions [1].

Diagnosis and follow-up in MS are usually based on assessment of clinical symptoms, in particular the presentation 
of relapses, and supported by magnetic resonance imaging (MRI). A disadvantage of MRI is its lack of specificity for a particular MS hallmark, as the detected lesions can be due to oedema, inflammation, gliosis, demyelination or axonal loss. In addition, current medical treatment aimed at delaying disease progression mainly targets the immune system. In this context, it is important to identify novel biomarkers for MS diagnosis and progression, as well as new therapeutic targets to reduce damage and improve disease recovery.

$\mathrm{S} 100 \mathrm{~B}$ is a small $\mathrm{Ca}^{2+}$-binding protein member of the $\mathrm{S} 100$ family, which is mostly expressed by astrocytes, a small subset of oligodendrocytes and certain neuronal subpopulations [2, 3]. S100B exerts both intracellular and extracellular functions. Intracellularly, S100B acts as a signalling molecule, promoting neuronal proliferation, oligodendrocyte differentiation and assembly of cytoskeleton components important for maintaining astrocyte morphology, while facilitating astrocyte and microglia migration [4]. Interestingly, regarding extracellular functions, S100B can either act as a neurotrophic or neurotoxic molecule, depending on the concentration attained. At low and physiological concentrations (nanomolar), S100B is thought to promote neurite extension and neuronal survival during development, enhance astrocytic proliferation, and favour microglia chemotactic ability and quiescence [5-8].

Under stress conditions, namely, traumatic brain injury [9] or CNS infection [10], S100B reaches concentrations in the micromolar range and exerts neurotoxic effects [11]. These effects include microglial and astrocyte activation, with release of inflammatory and oxidative stress mediators [12], which contribute to neuronal death [12, 13]. Both trophic and toxic effects of extracellular S100B are mediated in the brain by its binding to the receptor for advanced glycation end products (RAGE) [14].

Augmented S100B levels were first detected in cerebrospinal fluid (CSF) of MS patients in the acute phase [15]. More recently, Petzold and collaborators showed the presence of $\mathrm{S} 100 \mathrm{~B}$ in acute lesions of post-mortem brain tissue of patients with relapsing-remitting multiple sclerosis (RRMS) [16], while it was shown to be increased in CSF [17] or serum of MS patients, decreasing after immunosuppressive [18] or natalizumab [19] therapies. However, no further studies clarified the role of S100B and its receptor RAGE in different stages of MS lesions or on disease progression.

So, here, we aimed to evaluate the contribution of S100B as a biomarker of MS diagnosis and as a determinant of demyelination or delayed remyelination. Our findings in human CSF samples from RRMS patients showed a significant increase of S100B production at the time of diagnosis that was corroborated by a slight S100B increase in respective serum samples. Moreover, we showed that S100B is highly upregulated in active and chronic active MS lesions mainly in astrocytes. Enhanced expression of S100B receptor RAGE was predominantly observed in macrophages/microglia in active lesions. Using an ex vivo demyelinating model, we demonstrate that $\mathrm{S} 100 \mathrm{~B}$ is highly expressed and released upon demyelination, in parallel with activation of astrocytes and microglia as well as upregulation of pro-inflammatory cytokine and inflammasome-related gene expression. Interestingly, neutralization of extracellular S100B prevented demyelination, decreased reactive gliosis and abrogated expression of key pro-inflammatory factors. Overall, our data demonstrate that S100B expression is altered in MS patients, that the protein is involved in demyelination mechanisms and is crucial for the inflammatory milieu, which can be important for the design of new therapeutic strategies to reduce damage or promote tissue repair following MS episodes.

\section{Material and Methods}

\section{CSF and Serum Samples}

Patients with RRMS (all fulfilling revised McDonald 2005 criteria) were recruited at Hospital de Braga. The study was approved by the local ethics committee (CESHB) and the national authority for data protection (CNPD), and all participants gave written informed consent before inclusion. In this study, we included eleven patients at the time of diagnosis of RRMS and eleven controls (non-inflammatory/inflammatory neurological disorders). Detailed clinical data of MS patients and controls are summarized in Table 1. Between 3 and $5 \mathrm{~mL}$ of CSF were collected by lumbar puncture, and the first $2 \mathrm{~mL}$ were discarded/ used for clinical purposes, while the remaining was kept refrigerated until aliquoted and stored at $-80^{\circ} \mathrm{C}$ within $2 \mathrm{~h}$ of collection. Blood was also collected at the time of lumbar punctures, from a peripheral vein directly to serum tubes (4 mL). After clotting at room temperature, the serum

Table 1 Clinical data of patients with relapsing-remitting multiple sclerosis (RRMS) and non-inflammatory/inflammatory neurological disorder (NA) controls

\begin{tabular}{llllll}
\hline Case & Age & Sex & Case & Age & Sex \\
\hline NA 13 & 57 & M & RRMS 21 & 55 & M \\
NA18 & 25 & M & RRMS 33 & 22 & F \\
NA25 & 38 & F & RRMS 60 & 21 & F \\
NA31 & 39 & M & RRMS 61 & 26 & M \\
NA36 & 44 & M & RRMS 80 & 47 & M \\
NA58 & 59 & F & RRMS 82 & 27 & F \\
NA63 & 74 & M & RRMS 92 & 39 & M \\
NA65 & 84 & F & RRMS 104 & 25 & F \\
NA69 & 20 & F & RRMS 107 & 48 & F \\
NA78 & 30 & M & RRMS 109 & 38 & F \\
NA105 & 69 & F & RRMS 114 & 23 & M \\
\hline
\end{tabular}

$M$ male, $F$ female 
supernatant was also aliquoted (within $2 \mathrm{~h}$ of collection) and stored at $-80{ }^{\circ} \mathrm{C}$ until further use.

\section{S100B Determination}

Determination of S100B concentration was performed by inhouse enzyme-linked immunosorbent assay (ELISA) as usual in our laboratory [20]. Briefly, CSF and serum samples were incubated for $2 \mathrm{~h}$ at $37^{\circ} \mathrm{C}$ on a 96-well plate previously coated with a monoclonal anti-S100B antibody (1:1000, Sigma-Aldrich, St. Louis, MO, USA). Thereafter, a polyclonal antiS100B antibody (1:5000, DAKO, Glostrup, Denmark) was added and samples additionally incubated for $30 \mathrm{~min}$ at $37{ }^{\circ} \mathrm{C}$. Finally, an anti-rabbit peroxidase-conjugated antibody (1:5000, Santa Cruz Biotechnology, Santa Cruz, CA, USA) was added for further $30 \mathrm{~min}$ at $37{ }^{\circ} \mathrm{C}$. The colorimetric reaction with Sigma Fast OPD tablets ${ }^{\circledR}$ (Sigma-Aldrich) was measured at $492 \mathrm{~nm}$ in a microplate absorbance spectrophotometer.

\section{Brain Tissue}

Brain tissue was obtained in collaboration with the Department of Pathology, VU University Medical Center Amsterdam and the Netherlands Brain Bank, Amsterdam, the Netherlands. For immunohistochemical analysis, we selected brain samples from nine MS patients and two non-neurological controls, which were carefully matched for age, sex, and port-mortem delay. The Netherlands Brain Bank received permission to perform autopsies for the use of tissue and for access to medical records for research purposes from the ethics committee of the VU Medical Center (Amsterdam, the Netherlands). Tissue samples from control cases were taken from the subcortical white matter or corpus callosum. MS tissue samples were selected on the basis of post-mortem MRI and lesions were classified according to standard histopathological criteria as previously published [21]. Based on this classification, six active and six chronic active lesions were identified. Detailed clinical data of MS patients and controls are summarized in Table 2. Immediately after excision, tissue was fixed in formaldehyde and snapfrozen in liquid nitrogen for immunohistochemistry. The study was approved by the institutional ethics review board (VU University Medical Center, Amsterdam, the Netherlands), and all donors or their next of kin provided written informed consent from brain autopsy, use of material and clinical information for research purposes.

\section{Immunohistochemistry}

Frozen sections were stained as previously described [22, 23]. In short, 5- $\mu \mathrm{m}$-thick cryosections were collected on Superfrost Plus glass slides, defrosted at room temperature and fixed in acetone for $10 \mathrm{~min}$. After fixation and blocking
Table 2 Clinical data of patients with multiple sclerosis (MS) and nonneurological controls

\begin{tabular}{llllll}
\hline Case & Age & $\begin{array}{l}\text { Type } \\
\text { of MS }\end{array}$ & Gender & $\begin{array}{l}\text { Post-mortem } \\
\text { delay (h:min) }\end{array}$ & $\begin{array}{l}\text { Disease duration } \\
\text { (years) }\end{array}$ \\
\hline MS1 & 66 & SP & F & 6 & 22 \\
MS2 & 61 & SP & M & $9: 15$ & 31 \\
MS3 & 41 & PP & M & $7: 23$ & 14 \\
MS4 & 49 & SP & M & 8 & 25 \\
MS5 & 76 & PP & M & $7: 30$ & 26 \\
MS6 & 51 & SP & M & 11 & $>10$ \\
MS7 & 44 & ND & M & $10: 10$ & 21 \\
MS8 & 47 & ND & F & $4: 25$ & 21 \\
MS9 & 44 & ND & M & 12 & 16 \\
Ctr11 & 84 & NA & F & $6: 55$ & NA \\
Ctr12 & 56 & NA & M & $9: 15$ & NA \\
\hline
\end{tabular}

$S P$ secondary progressive MS, $P P$ primary progressive $\mathrm{MS}, N D$ not determined, $M$ male, $F$ female

[1\% bovine serum albumin (BSA), $0.05 \%$ Tween-20 and $10 \%$ goat serum in phosphate-buffered saline solution (PBS)], sections were incubated with primary antibodies for $1 \mathrm{~h}$ at room temperature. The following antibodies were used: proteolipid protein (PLP; 1:3000, Serotec, Raleigh, NC, USA) for myelin, HLA-DR major histocompatibility complex (MHC-II) clone LN3 (LN3; 1:1000) for macrophages/microglia, S100B (1:7000, Abcam, Cambridge, UK) and RAGE (1:200, Abcam). Detection was performed with EnVision Kit rabbit/mouse-labelled horseradish peroxidase (DAKO) for $30 \mathrm{~min}$ at room temperature. After a short rinse in tap water, sections were counterstained with haematoxylin for $1 \mathrm{~min}$ and extensively washed with tap water for $5 \mathrm{~min}$. Finally, sections were dehydrated within a series of ethanol and xylene baths and mounted with Entellan (Merck Millipore, Darmstadt, Germany). Images were taken on a Leica DM4000B microscope (Leica Microsystems Heidelberg GmbH, Mannheim, Germany).

\section{Immunofluorescence}

To reveal the cellular localization of S100B and RAGE, immunofluorescent double labelling was performed as described before [23]. Sections were incubated for $30 \mathrm{~min}$ in PBS containing $1 \%$ BSA, $0.05 \%$ Tween-20 and $10 \%$ normal goat serum. The sections were then incubated with glial fibrillary acidic protein (GFAP; 1:2000, Chemicon, Temecula, CA, USA) and S100B (1:3500) or LN3 (1:500) and RAGE $(1: 100)$ overnight at $4{ }^{\circ} \mathrm{C}$. The next day, sections were incubated with secondary antibodies for $1 \mathrm{~h}$ at room temperature. To reduce autofluorescence, sections were counterstained with Sudan Black (0.3 \% in ethanol $70 \%$; Sigma). Finally, sections 
were stained with Hoechst (1:1000; Molecular Probes, Invitrogen, Carlsbad, CA, USA) to visualize nuclei and mounted with mounting medium (DAKO). Images were taken on a Leica DM6000 microscope (Leica Microsystems Heidelberg $\mathrm{GmbH})$.

\section{Ex vivo model of demyelination}

To study the role of S100B during a demyelinating event, we used cerebellar organotypic slice cultures treated with lysophosphatidylcholine (LPC) as previously described [24]. Parasagittal slices were obtained from cerebellum of CD1 mouse pups at post-natal day 10. Briefly, brains were removed, cerebellum and attached hindbrain were isolated in PBS, and 400- $\mu \mathrm{m}$ slices were obtained using a McIlwain tissue chopper and kept in an air-liquid interface system. Separated slices were placed in the upper chamber of a $0.4-\mu \mathrm{m}$ pore cell culture (BD Falcon, Lincoln Park, NJ, USA) in a number of four slices per insert. Cell culture inserts were maintained in six-well cell culture plates containing $1 \mathrm{~mL}$ of medium in the plate well at a $37^{\circ} \mathrm{C}$ and $5 \% \mathrm{CO}_{2}$ conditioned atmosphere. Slice culture media consisted of $50 \%$ minimal essential media (MEM, Gibco, Life Technologies, Inc., Grand Island, USA), $25 \%$ heat-inactivated horse serum (Gibco), $25 \%$ Earl's balanced salt solution (Gibco), $6.5 \mathrm{mg} / \mathrm{mL}$ glucose, $25 \mathrm{mM}$ 4-(2-hydroxyethyl)-1-piperazineethanesulfonic acid (HEPES) (Biochrom AG, Berlin, Germany), and $1 \%$ of both L-glutamine (Sigma-Aldrich) and penicillin/ streptomycin (Sigma-Aldrich). After 3 days in vitro (DIV), slice culture media were totally replaced by a serum-free medium consisting of $98 \%$ Neurobasal-A (Gibco) and $2 \%$ B-27 (Gibco), supplemented with $2 \mathrm{mM}$ L-glutamine, $36 \mathrm{mM}$ glucose, $1 \% \mathrm{U} / \mathrm{mL}$ penicillin/streptomycin and $25 \mathrm{mM}$ HEPES. Half media were replaced every day and slices were maintained for 7 DIV before treatment, to allow myelination and the clearance of debris. Following 7 DIV, slices were exposed to a demyelinating insult with LPC $(0.5 \mathrm{mg} / \mathrm{mL}$ in serum-free culture media). Following 18-h treatment with LPC, slices were transferred to serum-free media in which cultures were maintained up to $48 \mathrm{~h}[24,25]$.

In parallel experiments, to ascertain S100B role on demyelination and glial reactivity, slices were incubated with LPC in the presence or absence of anti-S100B antibody $(1: 1000$, Abcam). Additionally, slices were also treated with a nonspecific antibody, a goat anti-rabbit secondary antibody, in the presence of LPC to confirm whether the presence of an isotype would change LPC-induced response. Supernatants were collected before and after LPC treatment. Slices were collected at 9 DIV ( $48 \mathrm{~h}$ post-LPC) and either stored in TRIzol ${ }^{\circledR}$ reagent at $-20{ }^{\circ} \mathrm{C}$ for RNA extraction or fixed in $4 \%$ paraformaldehyde in PBS for $1 \mathrm{~h}$, rinsed in PBS and stored in PBS at $4{ }^{\circ} \mathrm{C}$ for immunohistochemistry assays.

\section{Semi-quantitative RT-PCR}

Total RNA was extracted from 9-DIV slices using the TRIzol $^{\circledR}$ reagent (Invitrogen) method, according to the manufacturer's instructions. RNA concentration was quantified using NanoDrop ND-100 Spectrophotometer (NanoDrop Technologies, Wilmington, DE, USA). Aliquots of $500 \mathrm{ng}$ of total RNA were reversely transcribed into complementary DNA (cDNA) using the RivertAid H Minus First Strand cDNA Synthesis Kit (Thermo Fisher Scientific, MA, USA), under the recommended conditions. Quantitative RT-PCR (qRT-PCR) was performed using $\beta$-actin as an endogenous control to normalize the expression level of S100B, myelin basic protein (MBP), PLP, first-line cytokines: tumour necrosis factor (TNF)- $\alpha$; interleukin (IL)- $1 \beta$ and IL-6; and inflammasome-related molecules: IL-18, high-mobility group box protein 1 (HMGB1) and NLRP3. The sequences used as primers are listed in the Table 3. qRT-PCR was performed on a real-time PCR detection system (Applied Biosystems 7300 Fast Real-Time PCR System, Applied Biosystems, Madrid, Spain) using an SYBR Green qPCR Master Mix (Thermo Fisher Scientific). The PCR was performed in eight-well strips with each sample performed in duplicate, and a no-template control was included for each amplification product. qRTPCR was performed under optimized conditions: $50{ }^{\circ} \mathrm{C}$ for $2 \mathrm{~min}, 95^{\circ} \mathrm{C}$ for $10 \mathrm{~min}$ followed by 40 cycles at $95{ }^{\circ} \mathrm{C}$ for $15 \mathrm{~s}$ and $62{ }^{\circ} \mathrm{C}$ for $1 \mathrm{~min}$. To verify the specificity of the amplification, a melt-curve analysis was performed, immediately after the amplification protocol $\left(95^{\circ} \mathrm{C}\right.$ for $15 \mathrm{~s}$, followed by $60^{\circ} \mathrm{C}$ for $30 \mathrm{~s}$ and $95^{\circ} \mathrm{C}$ for $15 \mathrm{~s}$ ). Non-specific products of PCR were not detected in any case. Relative messenger RNA (mRNA) concentrations were calculated using the Pfaffl modification of the $\Delta \Delta \mathrm{C}_{\mathrm{T}}$ equation [cycle number at which fluorescence passes the threshold level of detection $\left(C_{T}\right)$ ], taking into account the efficiency values of individual genes. The results were normalized to the housekeeping gene $\beta$-actin in the same sample and the initial amount of the template of each trial was determined as relative expression by the formula $2^{-\Delta \Delta C T} . \Delta C_{T}$ is the value obtained, for each sample, by performing the difference between the mean $C_{T}$ value of each gene of interest and the mean $C_{T}$ value of $\beta$-actin. $\Delta \Delta C_{T}$ of one sample is the difference between its $\Delta C_{T}$ value and the $\Delta C_{T}$ of the sample chosen as reference.

\section{Immunostaining Procedure}

After fixation, membranes containing tissue sections were cut from cell culture insert and incubated with blocking solution (1 nM HEPES, $2 \%$ heat-inactivated horse serum, $10 \%$ heatinactivated goat serum, $1 \%$ BSA and $0.25 \%$ Triton X-100 in Hank's balanced salt solution) for $3 \mathrm{~h}$ at room temperature. The sections were then incubated with primary antibodies diluted in blocking solution for $24 \mathrm{~h}$ at $4{ }^{\circ} \mathrm{C}$. The following 
Table 3 List of pairs of primers used for qRT-PCR assays

\begin{tabular}{lll}
\hline Gene & Sense & Anti-sense \\
\hline S100B & GAGAGAGGGTGACAAGCACAA & GGCCATAAACTCCTGGAAGTC \\
MBP & CCATCCAAGAAGACCCCACA & CCCCTGTCACCGCTAAAGAA \\
PLP & TGGCGACTACAAGACCACCA & GACACACCCGCTCCAAAGAA \\
TNF- $\alpha$ & TACTGAACTTCGGGGTGATTGGTCC & CAGCCTTGTCCCTTGAAGAGAACC \\
IL-1 $\beta$ & CAGGCTCCGAGATGAACAAC & GGTGGAGAGCTTTCAGCTCATA \\
IL-6 & CCGGAGAGGAGACTTCACAG & GGAAATTGGGGTAGGAAGGA \\
IL-18 & TGGTTCCATGCTTTCTGGACTCCT & TTCCTGGGCCAAGAGGAAGTG \\
HMGB1 & CTCAGAGAGGTGGAAGACCATGT & GGGATGTAGGTTTCATTTCTCTTTC \\
NRLP3 & TGCTCTTCACTGCTATCAAGCCCT & ACAAGCCTTTGCTCCAGACCCTAT \\
$\beta-$ Actin & GCTCCGGCATGTGCAA & AGGATCTTCATGAGGTAGT \\
\hline
\end{tabular}

All primers were purchased from Thermo Fisher Scientific, MA, USA

$H M G B 1$ high-mobility group box protein 1, IL interleukin, $M B P$ myelin basic protein, $P L P$ proteolipid protein, $T N F$ tumour necrosis factor antibodies were used: neurofilament medium (NF-200, 1:200, Novocastra, Wetzlar, Germany) for neuronal axons, NG2 (1:50, Merck Millipore) for oligodendrocyte precursor cells, MBP (1:50, Serotec) for mature OLs, GFAP (1:100, Novocastra) for astrocytes, ionized calcium-binding adapter molecule 1 (Iba-1, 1:250, WAKO) for microglia and S100B (1:500, Abcam). Then, slices were washed for three times for 15 min each with PBS with $0.01 \%$ Triton X-100 (PBS-T) before incubation for another $24 \mathrm{~h}$ at $4{ }^{\circ} \mathrm{C}$ with secondary antibody in blocking solution. Slices were washed for three times for 15 min each with PBS-T, incubated 3 min with DAPI (1:1000), washed for three times for 15 min each with PBS-T and mounted using Fluoromount-G (Southern Biotech, Birmingham, AL, USA) for confocal microscopy. The percentage of the area immunoreactive for each antibody was measured in images captured using a $\times 20 / 1.2$ (zoom) lens on a Confocal Point Scanning Microscope Zeiss LSM 510 META (Zeiss, Germany). Binary masks were defined using a cut-off intensity threshold value for each region of interest, which corresponds to a minimum intensity due to specific staining above background values. Then, the percentage of the area occupied by NF-200, MBP, GFAP, Iba-1 and S100B was measured automatically using ImageJ software in each cerebellum region. Regarding myelination, the percentage of myelinated fibres was obtained by the ratio between the area of co-localization of NF-200 and MBP and the total area occupied by NF-200. Results are given by averaging values determined in the separate microscopic fields from slices of different animals.

\section{Statistical Analysis}

All results are presented as mean \pm SEM. The difference between control and RRMS patient samples was determined by the Mann-Whitney test, while differences in slice cultures were analyzed by the two-tailed $t$ test performed on the basis of equal and unequal variance or by one-way ANOVA with Tukey post-test, using GraphPad PRISM 5.0 (GraphPad Software, San Diego, CA, USA), as appropriate. The $P$ values of $P<0.05, P<0.01$ and $P<0.001$ were considered as being statistically significant.

\section{Results}

\section{S100B Levels in the CSF and Serum of RRMS Patients Are Increased at the Time of Diagnosis}

We first assessed S100B levels in CSF and serum samples of RRMS patients $(n=11)$ at the time of diagnosis and controls $(n=13)$ by ELISA. As depicted in Fig. 1, S100B concentrations were markedly increased in the CSF of MS patients at time of diagnosis of RRMS when compared with controls (1.66 vs. $0.77 \mathrm{ng} / \mathrm{L}, P<0.01)$. Although in a smaller magnitude, $\mathrm{S} 100 \mathrm{~B}$ levels were also elevated in the serum of the same patients (1.51 vs. $1.25 \mathrm{ng} / \mathrm{L}, P<0.05)$, which corroborates previous findings $[16,18]$, showing that $\mathrm{S} 100 \mathrm{~B}$ may be a potential diagnosis biomarker of MS.

\section{S100B and RAGE Expression in Human Control Brain and Normal-Appearing White Matter}

$\mathrm{S} 100 \mathrm{~B}$ is known to be increased in homogenates of MS lesions [16]; however, the cellular source of S100B is unknown. Hence, we decided to evaluate S100B and its receptor RAGE expression in brain samples of MS patients and controls. S100B was barely detectable in normal-appearing white matter (NAWM), while RAGE immunoreactivity was predominantly localized to nuclei of glial cells (Supplementary Fig. 1). No differences were observed comparing the expression of S100B and RAGE in NAWM with control white matter samples (data not shown). 

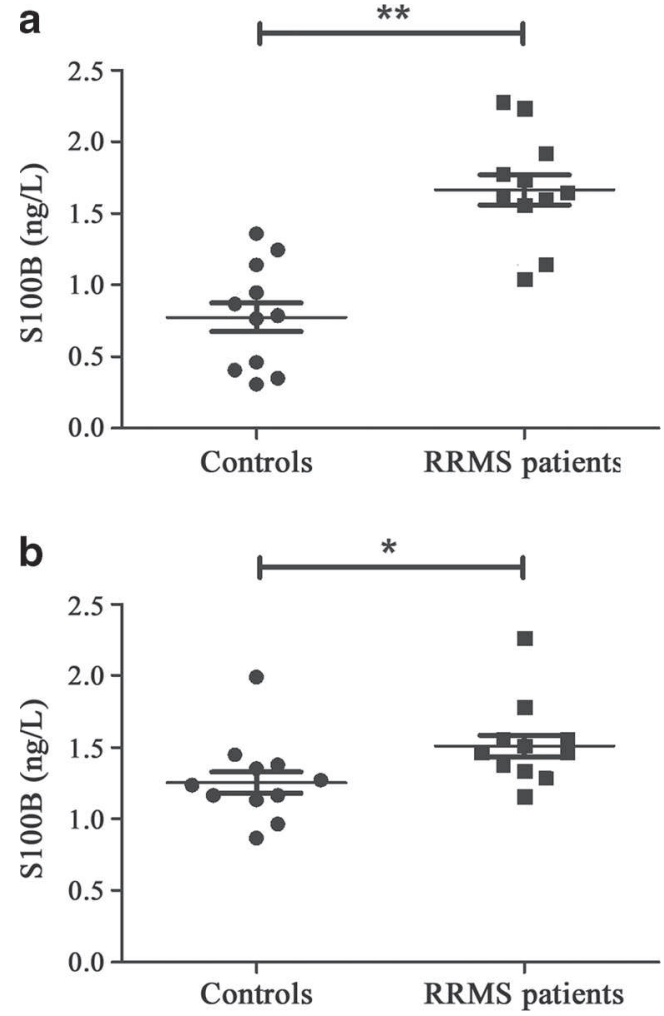

Fig. 1 S100B elevated levels detected in cerebrospinal fluid and serum of multiple sclerosis (MS) patients at time of diagnosis of relapsingremitting MS (RRMS) form. S100B was determined by ELISA in cerebrospinal fluid (a) and serum (b) of MS patients collected at time of diagnosis and of controls. Results are mean \pm SEM from eleven samples performed in duplicate. The Main-Whitney test was used to determine the statistical significance $\left({ }^{* *} P<0.01\right.$ and $* P<0.05$ vs. controls)

\section{S100B and RAGE Expression in MS Lesions}

Active demyelinating MS lesions are characterized by loss of myelin and abundant PLP-positive macrophages. S100B expression was markedly increased in demyelinated white matter regions (Fig. 2a) and localized to cell bodies and processes of reactive astrocyte-like cells (Fig. 2b). Astroglial source of S100B was further confirmed with double immunofluorescence labelling for S100B (green) and an astrocytic marker, GFAP (red) (inset in Fig. 2b-S100B). RAGE expression was also strikingly increased in active white matter lesions (Fig. 2a) and mainly localized to macrophages and activated microglia (Fig. 2b), which was confirmed by double immunofluorescence labelling for RAGE (green) and LN3 (red) (inset in Fig 2b-RAGE).

Analysis of chronic active MS lesions is characterized by a demyelinated lesion centre devoid of immune cells and a rim of activated microglia and macrophages. S100B expression was increased throughout the demyelinated areas (Fig. 3a). S100B diffuse staining resembles the morphology of astrocyte processes that constitute the gliotic scar tissue in the lesion centre (Fig. 3b). Conversely, RAGE was weakly expressed a

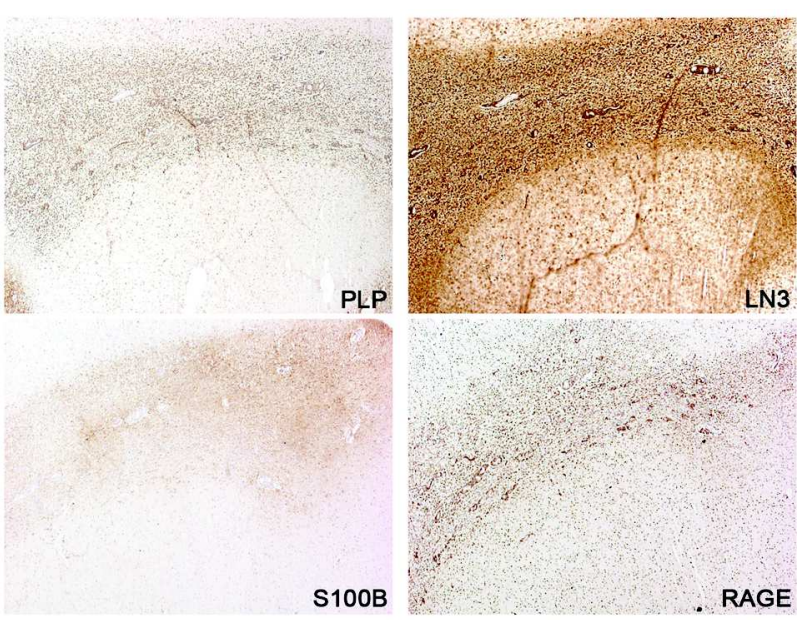

\section{b}

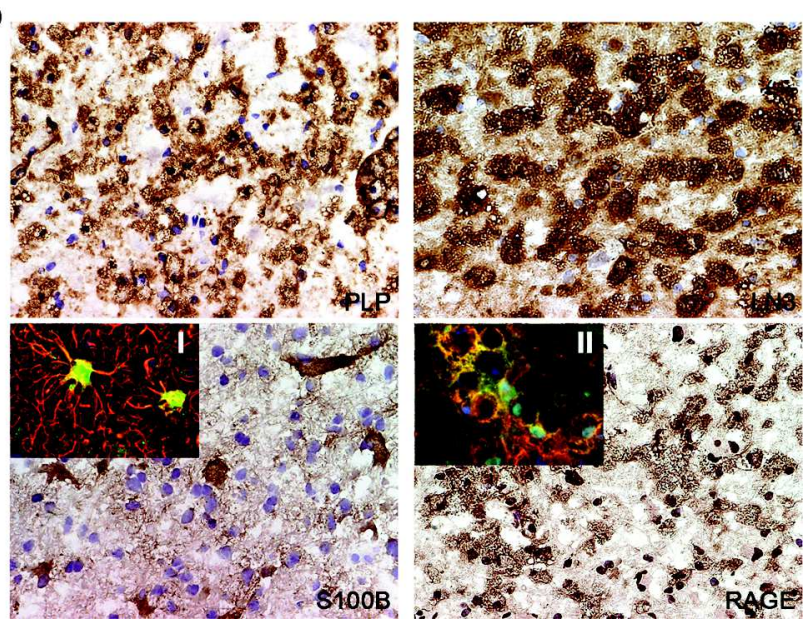

Fig. 2 S100B and its receptor, the receptor for advanced glycation end products (RAGE), are markedly expressed in active multiple sclerosis (MS) lesions by astrocytes and macrophages/microglial cells, respectively. Sequential frozen sections of autopsied brain samples of MS patients were immunostained for proteolipid protein $(P L P)$ to detect white matter and for HLA-DR MHC class II clone LN3 to detect macrophages/ microglial cells, as well as for S100B and RAGE. a S100B and RAGE expression is increased within active MS lesions, outlined by PLP staining and LN3 immunoreactivity. Magnification $\times 10$. b S100-positive cells have morphological characteristics of astrocytes and RAGE-positive cells of activated macrophages/microglia. Magnification $\times 40$. Insets show the co-localization of $(I)$ glial fibrillary acidic protein (GFAP, red), an astrocytic marker, with S100B (green) and the co-localization of (II) LN3 (red), a marker of activated microglia/macrophages with RAGE (green). Magnification $\times 63$

by a few cells in the rim but virtually absent in the inactive hypocellular centre (Fig. 3a, b).

\section{Ex vivo Demyelinating Model Shows a Marked Overexpression and Release of S100B Following the Demyelination Insult}

As we observed abnormal levels of S100B in CSF and serum of MS patients and in MS lesion samples, we decided to explore whether S100B was differentially expressed and 


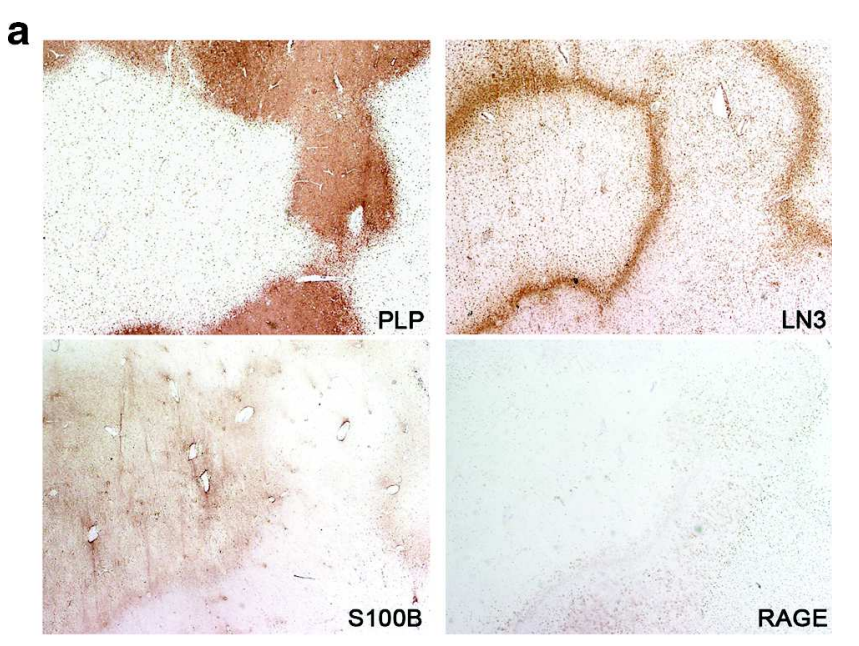

b

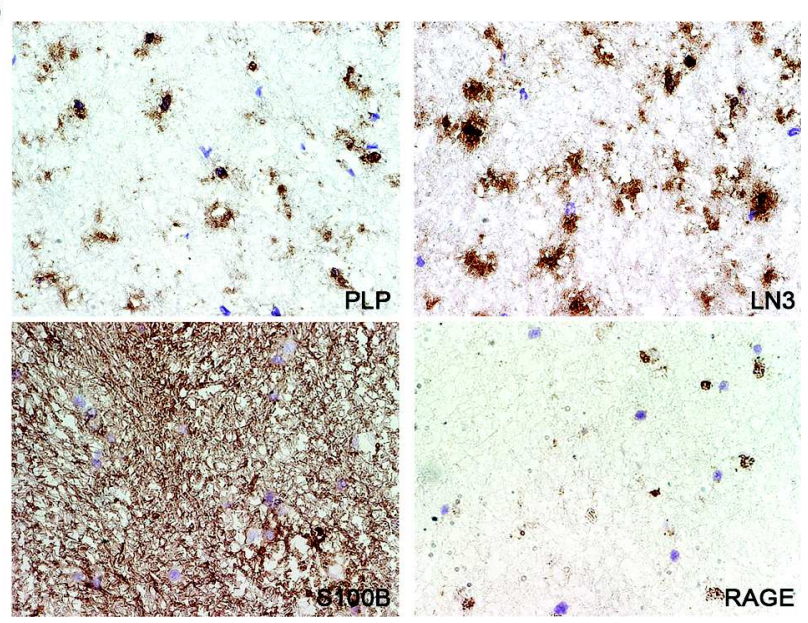

Fig. $3 \mathrm{~S} 100 \mathrm{~B}$ but not the receptor for advanced glycation end products $(R A G E)$ is continuously expressed in chronic multiple sclerosis (MS) lesions. Sequential frozen sections of autopsied brain samples of MS patients were immunostained for proteolipid protein $(P L P)$ to detect white matter and HLA-DR MHC class II clone LN3 to identify macrophages/ microglial cells, as well as for S100B and RAGE. a S100B is increased within the inactive centre of MS lesions and a weak RAGE expression is confined to the rim. Magnification $\times 10$. b Within the lesion, S100Bpositive cells have morphological characteristics of astrocytes, while only a few RAGE-positive cells are observed within the rim of the lesion. Magnification $\times 40$

secreted upon a demyelinating event. First, we examined if S100B was being expressed in our model of demyelination. In this context, the levels of $\mathrm{S} 100 \mathrm{~B}$ protein released to the extracellular space were quantified in slices or incubation media collected before the demyelination with LPC $(0 \mathrm{~h})$, immediately after the LPC stimulus of $18 \mathrm{~h}$, and at $48 \mathrm{~h}$, i.e. after $30 \mathrm{~h}$ of recovery, by ELISA. As shown in Fig. 4a, a striking increase in the release of S100B occurred upon $18 \mathrm{~h}$ of LPC incubation (20.8-fold vs. control, $P<0.001$ ), which was maintained at $48 \mathrm{~h}(12.3$-fold vs. control, $P<0.001)$. In addition, determination of S100B mRNA expression in the slices at $48 \mathrm{~h}$, by qRT-PCR, revealed that there was a significant increase of S100B expression (3.51-fold vs. control, $P<0.01$ ) even after $30 \mathrm{~h}$ of recovery post-LPC treatment, thus
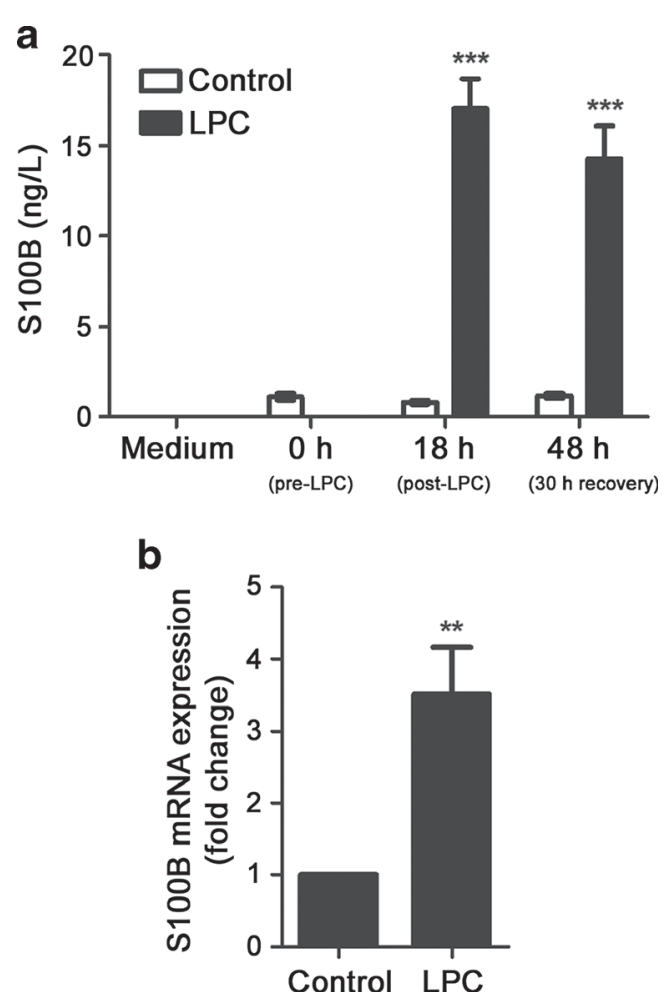

Fig. 4 Demyelination induces a massive release and continuous overexpression of S100B in cerebellar organotypic slice cultures. Cerebellar organotypic slice cultures were exposed to lysophosphatidylcholine $(L P C)$ at 7 days in vitro $(0 \mathrm{~h})$ for $18 \mathrm{~h}$. a Samples for detection of $\mathrm{S} 100 \mathrm{~B}$ secretion were collected before the incubation $(0 \mathrm{~h})$, at $18 \mathrm{~h}$ post-incubation with LPC and at $48 \mathrm{~h}$, i.e. after $30 \mathrm{~h}$ of recovery. b Samples for analysis of mRNA expression were collected at $48 \mathrm{~h}$. Results are mean \pm SEM from at least eight independent experiments. One-way ANOVA with Tukey post-test or $t$ test was used to determine the statistical significance as appropriate $(* * P<0.01$ and $* * * P<0.001$ vs. control)

suggesting that $\mathrm{S} 100 \mathrm{~B}$ is continuously induced by events resulting from the demyelinating insult (Fig. 4b).

\section{S100B Is Mainly Released by Astrocytes Following LPC Demyelination}

Knowing that S100B is mainly expressed by astrocytes in active MS lesions and that it is overexpressed and released to the extracellular space in our ex vivo demyelinating model, we further assessed the cellular origin of S100B in our experimental model. For this end, we performed double immunofluorescence to determine the cellular localization of S100B. Here, slices were double immunostained with antibodies against S100B and specific cellular markers (NF-200, GFAP or MBP). As shown in Fig. 5, S100B clearly co-localized with GFAP-positive astrocytes in control samples. Upon LPC treatment, co-localization of S100B with GFAP is the most prevalent, although we could observe some co-localization of S100B with MBP-positive structures. These findings corroborate the 
Fig. 5 S100B is mainly expressed by astrocytes both in control cultures and upon demyelination. Cerebellar organotypic slice cultures were exposed to

lysophosphatidylcholine $(L P C)$ at 7 days in vitro $(0 \mathrm{~h})$ for $18 \mathrm{~h}$. Immunostainings were performed in slices fixed at $48 \mathrm{~h}$ for $\mathrm{S} 100 \mathrm{~B}$, neurons [neurofilament-200 $(N F-200)$ ], astrocytes [glial fibrillary acidic protein $(G F A P)]$ and oligodendrocytes [myelin basic protein $(M B P)]$. S100B expression mainly co-localizes with GFAP-positive astrocytes both in control and in LPC treatment. Representative images of at least eight independent experiments are shown. Scale bar represents $100 \mu \mathrm{m}$

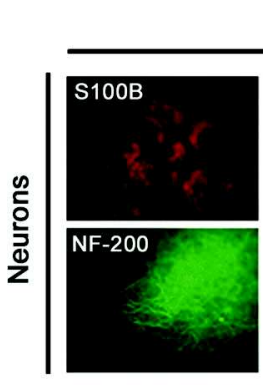

\section{Control}
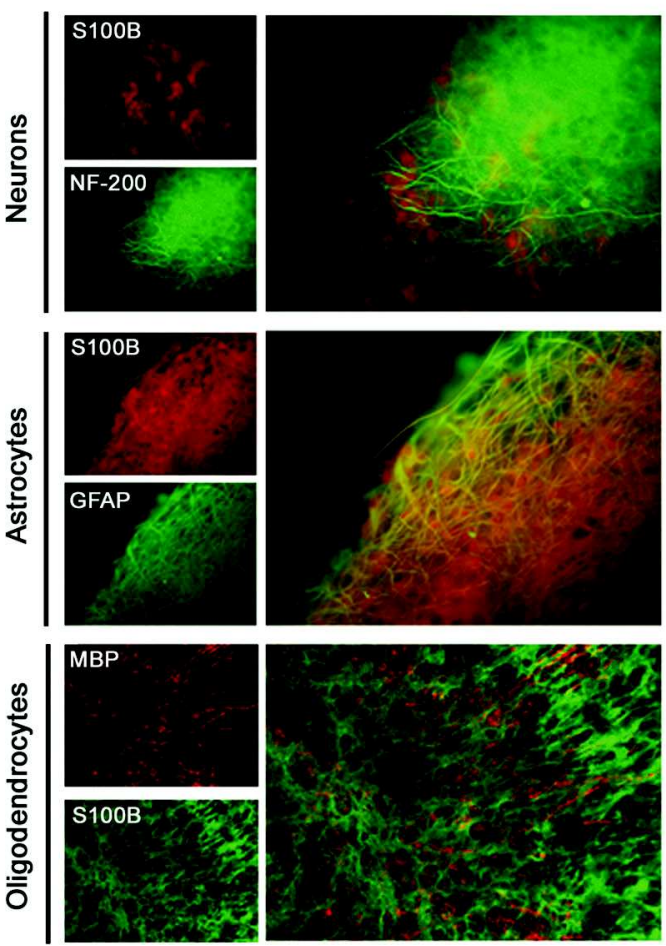

LPC
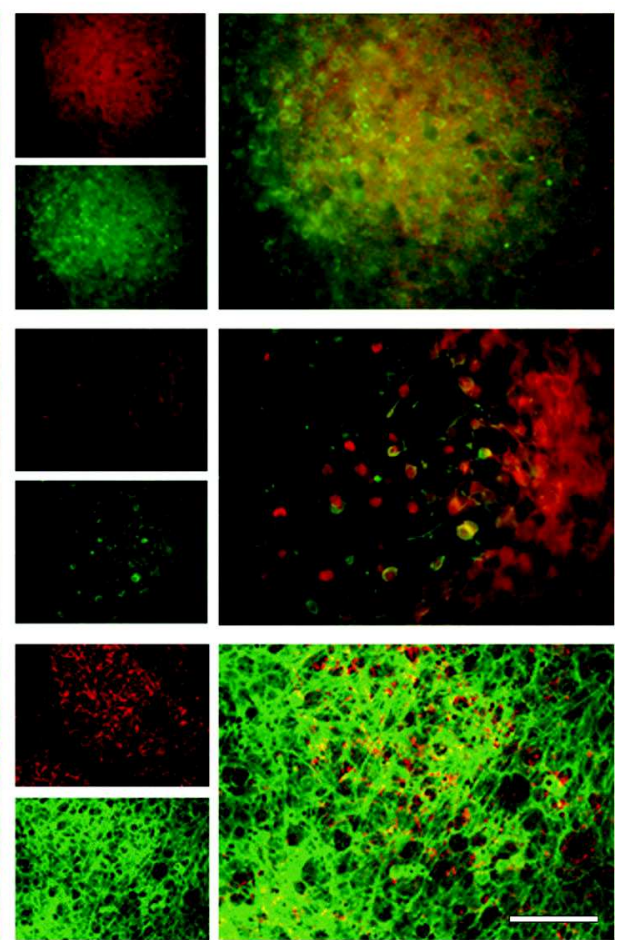

MS lesion data, indicating that astrocytes are the major producers of S100B under demyelinating circumstances.

\section{Neutralization of S100B Prevents LPC-Induced Demyelination}

Knowing that $\mathrm{S} 100 \mathrm{~B}$ is being overly secreted in response to LPC-induced demyelination, we wanted to understand whether the increase in extracellular S100B could be involved in the demyelination process. First, we measure the amount of NF200-positive axons to assure that LPC treatment was only affecting oligodendrocytes/myelin without axonal loss. As shown in Fig. 6a, b, the amount of NF-200 did not change following LPC incubation corroborating a specific oligodendrocyte/ myelin toxicity. Next, we evaluated the percentage of myelinated fibres in slices after recovery, which was calculated by the ratio of the area of co-localization of NF-200 and MBP and the area occupied by NF-200 alone. As depicted in Fig. 6a, c, LPC stimulus effectively damaged myelin sheaths, which was corroborated by the decrease observed in the percentage of myelinated fibres ( 0.60 -fold, $P<0.01)$. Interestingly, co-incubation with anti-S100B antibody prevented the demyelination caused by LPC in $55 \%(P<0.01)$. To assure that the use of an antibody did not have any role on the prevention of demyelination, we also evaluated the gene expression of MBP and PLP in cultures treated with a non-specific IgG plus LPC in parallel with the presented incubation scheme. As shown in Supplementary Fig. 2, LPC incubation markedly affected MBP and PLP
mRNA expression (0.61-fold, $P<0.01$, and 0.70 -fold, $P<0.05$, respectively) that was partially prevented by $\mathrm{S} 100 \mathrm{~B}$ neutralization (59\%,P<0.05, and $37 \%$, respectively). Coincubation of LPC with IgG did not change MBP and PLP mRNA expression corroborating that the presence of an isotype does not alter LPC-induced response. These findings indicate that S100B plays a role in LPC-dependent demyelination of cerebellar organotypic slice cultures.

\section{Abrogation of S100B Decreases Astroglial Reactivity Induced by Demyelination}

Along with myelin degeneration, demyelinating lesions are also characterized by astrocytosis and microgliosis [26, 27]. Regarding this issue and knowing that S100B is mainly secreted by astrocytes and is involved in the activation of both astrocytes and microglia, we decided to evaluate the degree of reactive gliosis in the course of the demyelinating insult with LPC (Figs. 7 and 8). LPC-treated slices evaluated at $48 \mathrm{~h}$ showed a marked decrease in the percentage of area occupied by astrocytes ( 0.4 -fold vs. control, $P<0.01)$, as a consequence of reduced extension and number of cellular processes in activated cells (Fig. 7). Interestingly, when slices were coincubated with the neutralizing S100B antibody, astrocytes retained their more ramified morphology as seen in control slices, increasing the percentage of the area occupied by astrocytes (1.2-fold vs. control), thus suggesting a reduced activated state. 
Fig. 6 Antibody-directed neutralization of S100B prevents demyelination caused by lysophosphatidylcholine $(L P C)$. Cerebellar organotypic slice cultures were exposed to LPC at 7 days in vitro for $18 \mathrm{~h}$. Double immunostainings were performed in slices fixed at $48 \mathrm{~h}$ for neuronal axons [neurofilament-200 $(N F-200)]$ and mature oligodendrocytes [myelin basic protein $(M B P)]$ and nuclei were stained with DAPI (blue). a Representative images are shown. Scale bar represents $100 \mu \mathrm{m}$. b Quantification of axon integrity was taken by averaging the area occupied by NF-200 staining for each stack. c The percentage of myelinated fibres was calculated by the ratio between the area of co-localization of NF-200 and MBP and the total area occupied by NF-200. Results are mean \pm SEM from at least eight independent experiments. One-way ANOVA with Tukey post-test was used to determine the statistical significance as appropriate $(* * P<0.01$ vs. control; \#\#P<0.01 vs. LPC alone) a

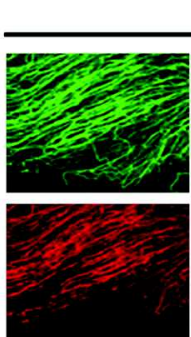

Control
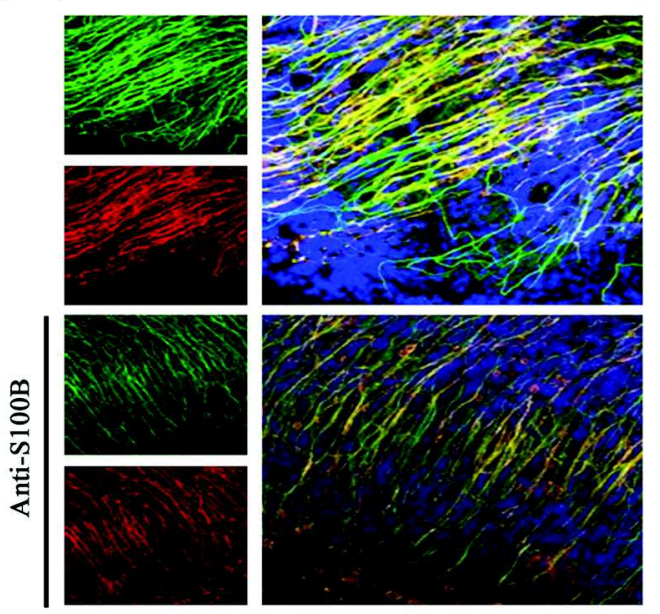

NF-200 $\square$ MBP $\square$ DAPI

b

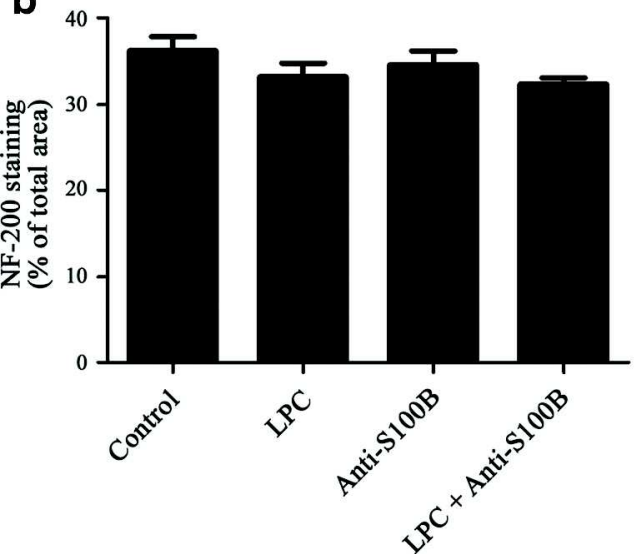

LPC
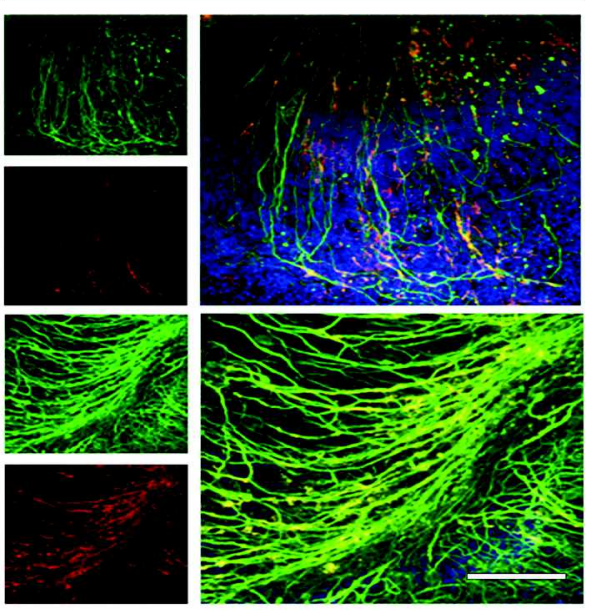

C

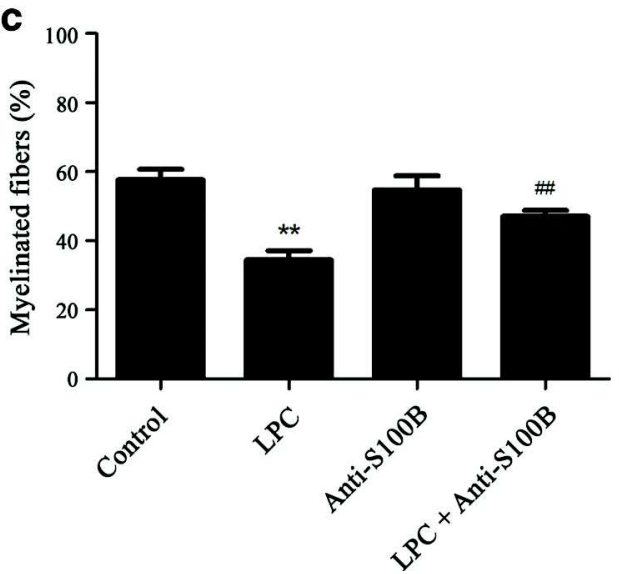

Regarding microglia, as shown in Fig. 8, incubation with LPC markedly increased the area occupied by Iba-1-positive microglia (2.0-fold vs. control, $P<0.01)$, suggesting an increase of microglial number, and changed their morphology to a more amoeboid/activated state. Co-incubation with antiS100B antibody in the presence of LPC did not attenuate microglial proliferation/activation induced by LPC-mediated demyelination.

\section{Neutralization of S100B Prevents LPC-Induced Gene Expression of First-Line Cytokines and Inflammasome-Associated Components}

Along with astrocytic and microglial activation, demyelination is accompanied by exacerbated production of proinflammatory cytokines and an increased production of inflammasome-related proteins $[19,28]$. This inflammatory milieu is crucial for determination of lesion extent, immune cell recruitment and ability to remyelinate. Curiously, S100B was reported to promote microglial and astroglial release of IL- $1 \beta$ and TNF- $\alpha$ when present in elevated concentrations [12], as we have observed here in our demyelinating model.
So, we then evaluated gene expression of these inflammatory mediators upon demyelination and in the presence of antiS100B antibody. As depicted in Fig. 9, there is still a marked increase in the expression of first-line cytokines TNF- $\alpha$ (2.2fold vs. control, $P<0.01)$ and IL-1 $\beta$ (6.8-fold vs. control, $P<0.01)$ and a reduction of IL-6 $(0.3$-fold vs. control, $P<0.01)$, during the recovery period after LPC insult. Interestingly, when slices exposed to LPC were co-incubated with anti-S100B antibody, the expression of both TNF- $\alpha$ and IL$1 \beta$ remained similar to control values $(P<0.01)$, while IL-6 inhibition was prevented by $\sim 50 \%(\mathrm{P}<0.01)$. These results corroborate the induction of a marked inflammatory response upon demyelination induction and suggest that S100B is involved in cytokine release by glial cells.

Inflammasomes are cytosolic protein complexes involved in the maturation and secretion of pro-inflammatory mediators including IL-1 $\beta$, IL-18 and HMGB1 [28, 29]. More recently, inflammasomes, namely, the NLRP3 inflammasome, have been associated with MS development [28]. Since we have observed such a marked LPC-induced increase of IL-1 $\beta$ and protection in the presence of anti-S100B, we next assessed alterations in gene expression of the other inflammasome- 
Fig. 7 S100B neutralization prevents astrocytic morphological activation in the course of demyelination. Cerebellar organotypic slice cultures were exposed to

lysophosphatidylcholine ( $L P C)$ at 7 days in vitro for $18 \mathrm{~h}$. Double immunostainings were performed in slices fixed at $48 \mathrm{~h}$ for astrocytes [glial fibrillary acidic protein $(G F A P)$ ] and mature oligodendrocytes [myelin basic protein $(M B P)]$. Nuclei were stained with DAPI (blue). a Representative images are shown. Scale bar represents $100 \mu \mathrm{m}$. b Quantification of astrocytes was taken by averaging the area occupied by GFAP staining for each stack. Results are mean \pm SEM from at least eight independent experiments. One-way ANOVA with Tukey post-test was used to determine the statistical significance as appropriate $(* * P<0.01$ vs. control; \#\#P<0.01 vs. LPC alone) a
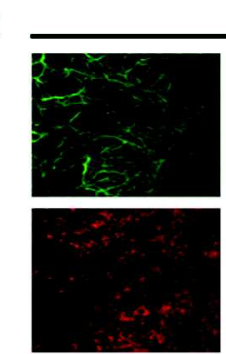

Control
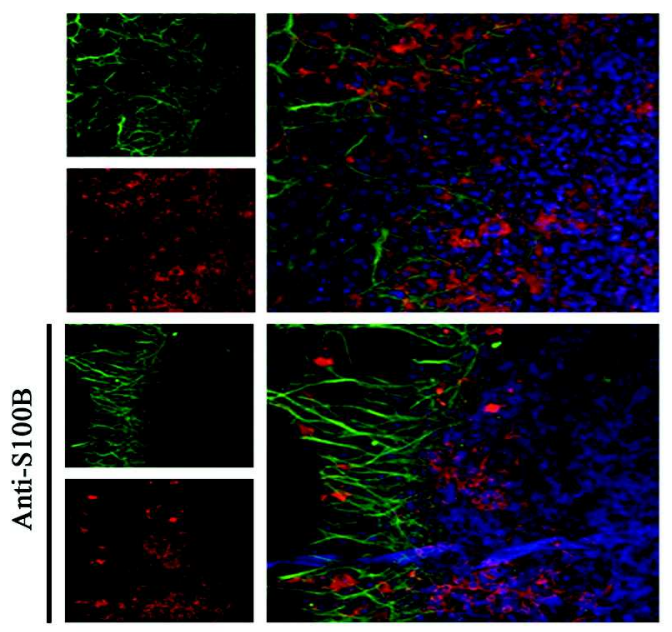

GFAP MBP $\square D A P I$
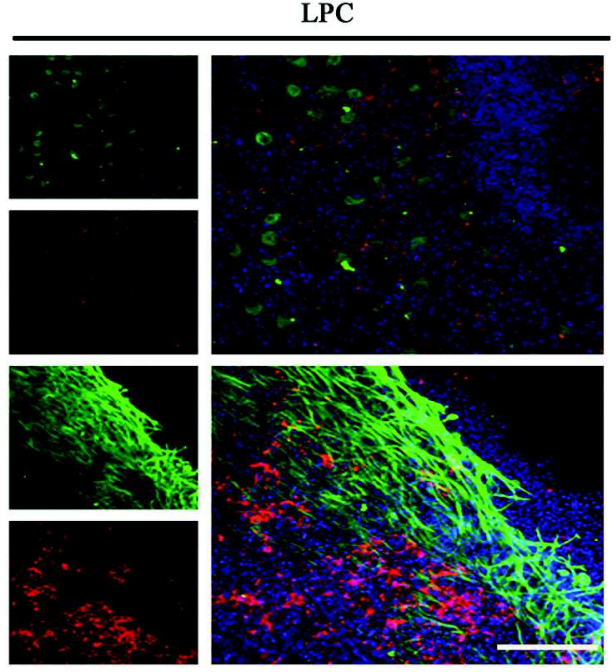

b

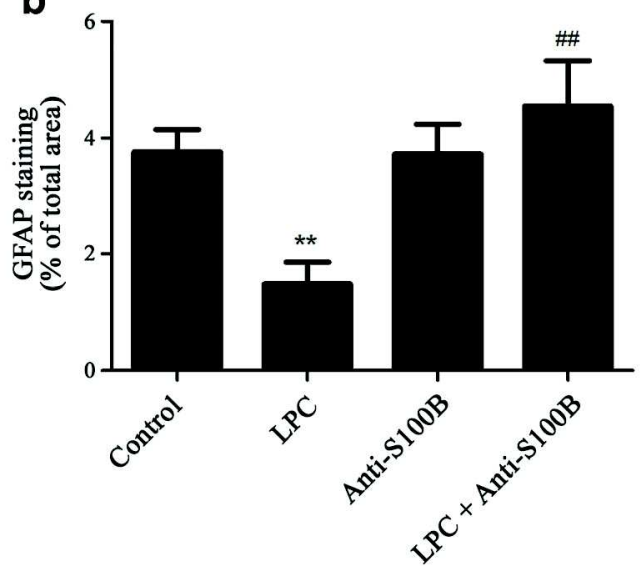

related molecules. As shown in Fig. 10, LPC-induced demyelination markedly increased IL-18 (10.9-fold vs. control, $P<0.01$ ), HMGB1 (14.8-fold vs. control, $P<0.01$ ) and NLRP3 (11.0-fold vs. control, $P<0.01)$ gene expression. As observed for cytokine profile, co-incubation of LPC-treated slices with anti-S100B antibody significantly inhibited the LPC-induced expression of HMGB1, IL-18 and NRLP3, in which levels remained similar to control ones $(P<0.01)$. These results clearly indicate that by neutralizing $\mathrm{S} 100 \mathrm{~B}$, we can reduce the inflammatory milieu during a demyelinating insult. Moreover, since microglia are the most potent producers of inflammatory cytokines, it is important to note that even if the number of microglia was not altered by S100B neutralization, their phenotype may have shifted from a neurotoxic to a more neuroprotective one.

\section{Discussion}

In the present study, we show that S100B levels are significantly increased in CSF and serum samples from MS patients at the time of diagnosis of RRMS and that S100B expression is strikingly upregulated in active and chronic active lesions where it is predominantly localized to reactive astrocytes. Moreover, we observed an increased expression of its receptor RAGE in macrophages/microglia throughout active lesions. Using an experimental demyelinating model, we demonstrated that demyelination induces a marked upregulation and release of S100B protein, in parallel with astrocytosis, microgliosis and enhanced gene expression of key proinflammatory cytokines and NLRP3 inflammasome molecules. Therapeutic antibody-mediated neutralization of S100B prevented LPC-induced demyelination, reactive astrogliosis and cytokine and inflammasome expression. Taken together, our data indicate that S100B is a key element in the inflammatory process of an MS lesion and an interesting therapeutic target.

Firstly, we showed that S100B levels were higher in the CSF and serum of MS patients at the time of diagnosis of RRMS when compared to control patients. Although increased CSF concentrations of S100B were already reported in previous studies in acute phases or during the course of the disease $[16,18,30,31]$, our study is the first to demonstrate 
Fig. 8 S100B neutralization does not prevent microglia proliferation/activation induced by lysophosphatidylcholine (LPC) demyelination. Cerebellar organotypic slice cultures were exposed to LPC at 7 days in vitro for $18 \mathrm{~h}$. Double

immunostainings were performed in slices fixed at $48 \mathrm{~h}$ for microglia [ionized calciumbinding adapter molecule- 1 (Iba-1)] and mature oligodendrocytes [myelin basic protein $(M B P)]$. Nuclei were stained with DAPI (blue). a Representative images are shown. Scale bar represents $100 \mu \mathrm{m}$. b Quantification of microglia was taken by averaging the area occupied by Iba-1 staining for each stack. Results are mean \pm SEM from at least eight independent experiments. One-way ANOVA with Tukey post-test was used to determine the statistical significance as appropriate $(* * P<0.01 \mathrm{vs}$. control)
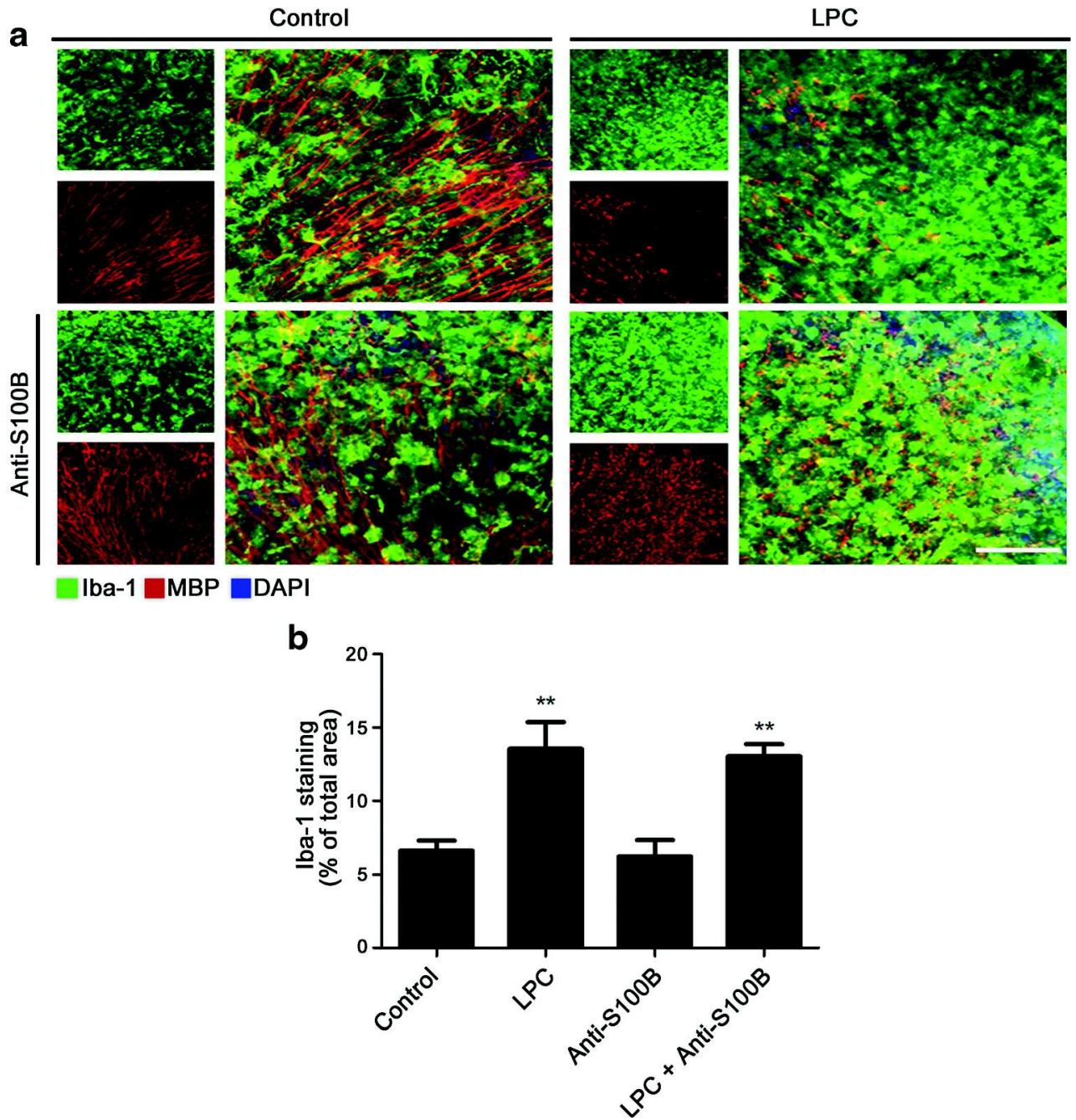

that also at the time of diagnosis, S100B can be viewed as an initial biomarker of MS. These results are also in line with a previous study from Petzold and colleagues demonstrating a significant trend for increasing S100B levels from primary progressive multiple sclerosis (PPMS) to secondary progressive multiple sclerosis (SPMS) to RRMS [16], suggesting its potential usefulness as a differential biomarker to distinguish between the different types of MS. Additional studies should, however, be performed to evaluate whether different levels of S100B could be associated with different MS stages and therefore be used as a prognostic tool. Moreover, since S100B was recently reported to decrease upon MS treatment with natalizumab but not with interferons, it can be also considered as a biomarker for treatment efficacy. Increased levels of S100B were similarly noticed in situations of acute brain damage including stroke [32], rapid parenchymal destruction [33] or traumatic brain injury [34]. However, this increase should be distinguished from that observed in progressive diseases, such as MS, where sustained S100B, as detected herein in the chronic MS lesions, might have different roles by modulating the inflammatory response $[35,36]$ and consequently modify the disease progression by yet unknown mechanisms. Although there are other sources of S100B than the CNS, such as adipose tissue, testis and skin [15, 37, 38], the elevation of $\mathrm{S} 100 \mathrm{~B}$ in serum has been associated with blood-brain barrier (BBB) disruption [39], which is in line with the presence of signs of BBB breakdown in the very early stages of MS [40].

Petzold and colleagues demonstrated increased levels of S100B in tissue homogenates of distinct lesion types [16]; however, information on the cellular source of S100B and localization of RAGE in the CNS has been mainly based on immunohistochemical studies using rodent brain samples. Here, we show for the first time that astrocytes in active and chronic active lesions abundantly express S100B. In fact, taking into account rodent data, astrocytes are assumed as the CNS cell type with the highest expression rate of S100B and to constitutively secrete the protein $[36,41]$. Concerning S100B receptor RAGE expression, in NAWM the receptor is predominantly found in the nuclei of glial cells, suggesting an immature/inactive state. Mature RAGE, composed of three 
a

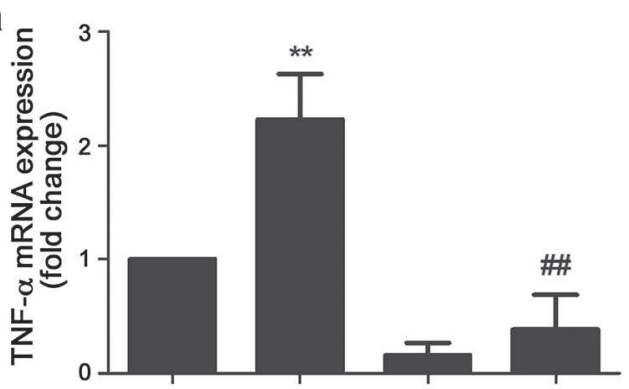

b

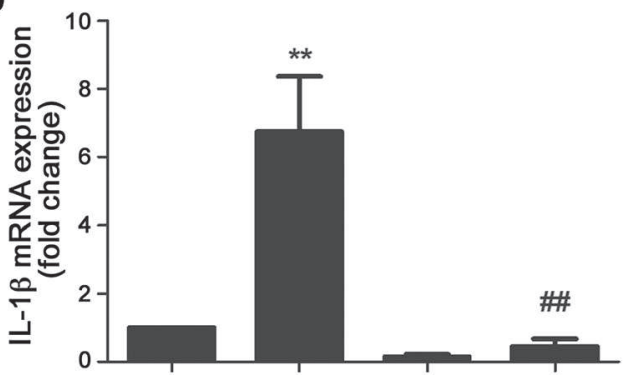

C

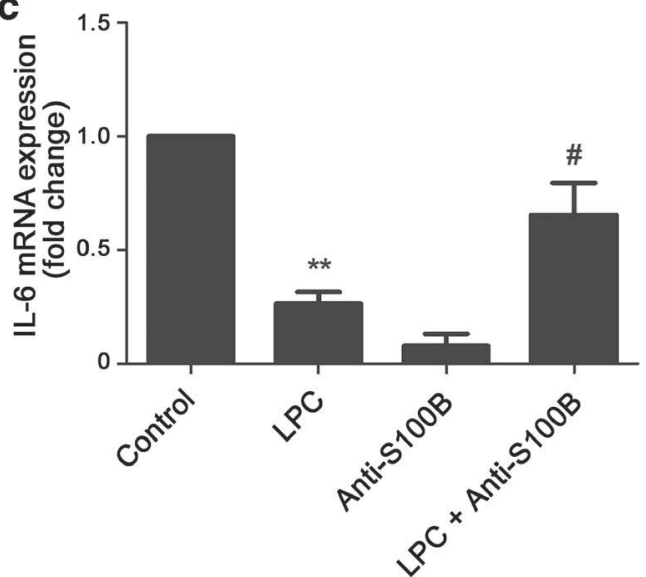

Fig. 9 S100B neutralization prevents the increase of tumour necrosis factor $(T N F-\alpha)$ and interleukin $(I L-1 \beta)$ expression and the inhibition of IL-6 expression induced by lysophosphatidylcholine $(L P C)$ demyelination. Cerebellar organotypic slice cultures were exposed to LPC at 7 days in vitro for $18 \mathrm{~h}$. Gene expression of TNF- $\alpha$ (a), IL-1 $\beta$ (b) and IL-6 (c) was assessed at $48 \mathrm{~h}$ by qreal-time PCR. Results are mean \pm SEM from at least eight independent experiments. One-way ANOVA with Tukey posttest was used to determine the statistical significance as appropriate ( $* * P<0.01$ vs. control; $\# P<0.05$ and $\# \# P<0.01$ vs. LPC alone)

major domains, an extracellular ligand-binding domain, a single transmembrane helix and a C-terminal domain [42], has to form constitutive multimers within the cytoplasmic membrane to become engaged and activate downstream signalling [43, 44]. In accordance, nuclear expression of RAGE in NAWM may represent a ubiquitous expression of RAGE under a nonactive form. Conversely, microglial and macrophage RAGE expression was enhanced in active lesions as well as in the rim of chronic active lesions, and its expression was within cell soma and processes suggesting RAGE assembly to the membrane allowing its subsequent engagement by S100B. S100B action through RAGE, a multiligand receptor, usually involves

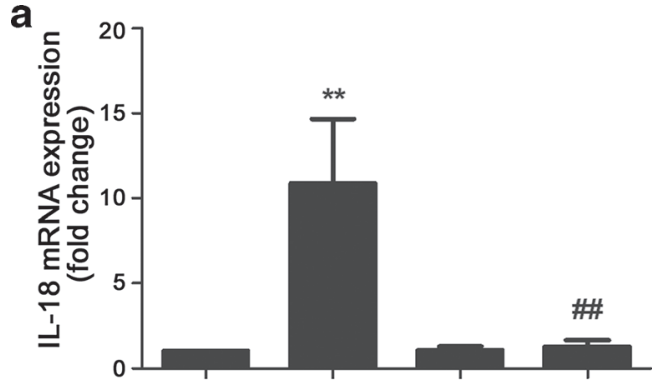

b
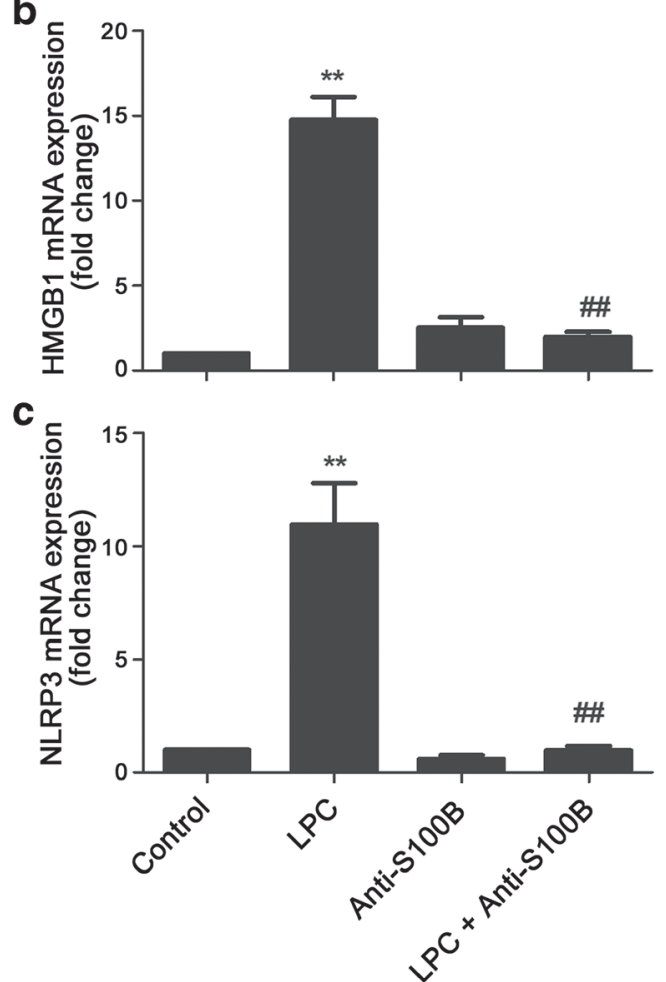

Fig. 10 S100B neutralization prevents NLRP3 activation and inflammasome-related protein expression induced by lysophosphatidylcholine $(L P C)$ demyelination. Cerebellar organotypic slice cultures were exposed to LPC at 7 days in vitro for $18 \mathrm{~h}$. Gene expression of interleukin (IL-18) (a), high-mobility group box chromosomal protein $1(H M G B 1)$ (b) and NRLP3 (c) was assessed at $48 \mathrm{~h}$ by qreal-time PCR. Results are mean \pm SEM from at least eight independent experiments. One-way ANOVA with Tukey post-test was used to determine the statistical significance as appropriate $(* * P<0.01$ vs. control; $\# \# P<0.01$ vs. LPC alone)

pro-inflammatory responses by activation of nuclear factor-kB, including the expression of cytokines [45] and recruitment of astrocytes [46] and microglia [47, 48] to the damaged site.

Using LPC-induced demyelination of cerebellar organotypic slice cultures, we observed that a demyelinating insult elicits a marked astrocytic upregulation and secretion of $\mathrm{S} 100 \mathrm{~B}$ indicating that astrocytes are the major producers of $\mathrm{S} 100 \mathrm{~B}$ in the course of demyelination. In agreement with this, recent studies in ex vivo rat cerebellar slice cultures have shown an increase in astrocyte population 2 DIV after a demyelination insult with LPC [25]. The minor co-localization 
with oligodendrocytes suggests that these cells are able to produce small amounts of S100B when exposed to a toxic stimulus, as previously described for an oligodendrocyte cell line, the OL-93, under serum and glucose deprivation conditions [49]. Most striking, we observed that sequestering excessive release of S100B significantly reduced LPC-mediated myelin loss, indicating that during demyelination the released $\mathrm{S} 100 \mathrm{~B}$ is promoting either directly or indirectly demyelination or delayed remyelination. It is known that high $\mathrm{S} 100 \mathrm{~B}$ triggers microglial and astrocyte activation promoting the release of nitric oxide (NO) and TNF- $\alpha$ [12], which are deleterious for oligodendrocytes [50] and may exacerbate demyelination. Curiously, our ongoing studies on the role of S100B on oligodendrocyte differentiation using primary cultures demonstrated that high $\mathrm{S} 100 \mathrm{~B}$, at the micromolar range, affects oligodendrocyte precursor cell differentiation and maturation into myelinating oligodendrocytes, while low physiological S100B levels slightly enhance oligodendrocyte maturation. These results suggest that during remyelination S100B high levels may directly affect this process possibly delaying de novo myelin formation. It deserves to be noted that expression of S100B by oligodendrocyte precursor cells is needed for a proper differentiation into myelinating oligodendrocytes [51], so by neutralizing the excessive S100B in the inflammatory milieu using a directed antibody, we are only acting extracellularly preventing S100B toxic effects. Conversely, a complete and non-targeted blockade of S100B expression using silencing or knock-down techniques may prove to be a deleterious approach affecting remyelination and damage recovery.

Reactive gliosis is a common pathological feature of MS pathology [52]. Our results show an increase of astrocytic activation upon LPC treatment evidenced by reduction of cell extension length and inflated cell body. This LPC-induced activation of astrocytes was shown to be diminished in the presence of anti-S100B. Reactive astrocytes, which are implicated in formation of the glial scar, are characterized by profound morphological and genetic changes [27]. Astrocyte activation has been observed during LPC-induced demyelination [25], and here, we show that astrocytes abundantly secrete $\mathrm{S} 100 \mathrm{~B}$ when exposed to the demyelinating agent. Moreover, excess of extracellular S100B levels may induce autocrine astrocytic activation that turns astrocytes into a proinflammatory and neurodegenerative phenotype [53]. In this situation, astrocytes secrete pro-inflammatory factors, which are known inhibitors of oligodendrocyte precursor cell proliferation and maturation, following demyelination in MS [54]. Thus, the apparent absence of astrocytosis under demyelinating conditions in the presence of anti-S100B is a potential indication that high levels of S100B might be favouring demyelination. Moreover, astrocytes are involved in the production of growth factors and chemokines that promote oligodendrocyte precursor cell activation and differentiation [55].
Therefore, dampening astrocyte activation by S100B antibody treatment may also boost remyelination.

Microglial activation contributes to a pro-inflammatory environment by the secretion of pro-inflammatory factors, potentiating demyelination, a feature that has also been described in ex vivo models as a consequence of demyelination $[25,56]$. Accordingly, our results reveal an increase in microglial proliferation and activation in the course of demyelinating insult which corroborates the increase of RAGE-expressing microglia/macrophages observed in lesion specimens. In addition, in parallel to increased S100B, we observed a marked release of first-line cytokines TNF- $\alpha$ and IL-1 $\beta$ upon demyelination, which corroborates previous studies showing that high S100B concentrations induce microglial secretion of pro-inflammatory cytokines $[4,35]$. On the other hand, our results show that neutralization of $\mathrm{S} 100 \mathrm{~B}$ does not change microglia number in the slice but prevents TNF- $\alpha$ and IL$1 \beta$ induction, as well as IL-6 inhibition following LPC treatment. Since these cytokines are mainly expressed by activated microglia, their inhibition following S100B neutralization corroborates microglial shift from a pro-inflammatory phenotype to a more neuroprotective one. Indeed, microglia have an important role in remyelination by clearing myelin debris [57, 58] and switching from a cytotoxic to protector phenotype at remyelination initiation [59]. Moreover, once extracellular $\mathrm{S} 100 \mathrm{~B}$ is known to inhibit microglial activation at low concentrations $[4,35]$, it is possible that $\mathrm{S} 100 \mathrm{~B}$ neutralization can prevent microglial pro-inflammatory activation upon demyelination, a finding beyond the scope of this manuscript.

Several lines of evidence suggest the involvement of NLRP3 inflammasome on MS development. Indeed, not only IL-1 $\beta$ [60] but also HMGB1 [61] and IL-18 [62] were found to be upregulated either in the serum, CSF or active lesions of MS patients or in rodent experimental autoimmune encephalomyelitis (EAE) lesions and associated with disease progression [63, 64]. Also, NLRP3 has been associated with MS progression and it was recently reported that Nlrp3 KO mice are resistant to EAE [65]. Our ex vivo demyelinating model shows increased expression of HMGB1, IL-1 $\beta$ and IL-18, thus corroborating such findings. Most attractively, the marked inhibition of inflammasome-related molecules by neutralization of S100B shows for the first time the involvement of S100B in NLRP3 inflammasome induction upon demyelination. Curiously, NLRP3 expression [66], as well as RAGE activation [67], was recently associated with chemotactic immune cell migration to the CNS in EAE, a hallmark of disease progression and damage exacerbation. So, our findings also suggest that S100B either alone or through NLRP3 inflammasome induction may be promoting CNS immune cell invasion during demyelination, which may be potentially attenuated by S100B therapeutic neutralization as tested here.

Taking into account the beneficial outcome of S100B inhibition in our ex vivo demyelinating model, it seems that this 
protein may be considered a potential therapeutic target to reduce damage during MS course. S100B targeting has already been tested in other disease models with promising results. In fact, pentamidine, a S100B inhibitor, reduces S100B and RAGE expression in an animal model of Alzheimer's disease, with consequent reduction of pro-inflammatory milieu in the hippocampus [68]. Other potential therapeutic strategies may be the use of specific RAGE antibodies to prevent S100B binding, small molecules or anti-S100B aptamers [69, 70]. In an indirect manner, also, induction of immune tolerance, as described for the anterior-chamber-associated immune deviation (ACAID) method [71], by preventing myelin destruction, could avoid astrocyte activation and consequent $\mathrm{S} 100 \mathrm{~B}$ release.

Taken together, the high production of S100B at the time of diagnosis of RRMS and its presence in active and chronic active MS lesions suggest its interest as a potential new biomarker for MS diagnosis. Moreover, based on the beneficial outcome of its inhibition in an ex vivo demyelinating model, S100B may also be considered a potential therapeutic target to reduce damage during the course of MS.

Acknowledgments This work was supported by Medal of Honor L'Oréal for Women in Science (FCT, UNESCO, L'Óreal) and innovation grant (Ordem dos Farmacêuticos) to AF, a post-doctoral grant from Fundação para a Ciência e Tecnologia (FCT-SFRH/BPD/96794/2013) and a DuPré Grant from the European Committee for Treatment and Research in Multiple Sclerosis (ECTRIMS) to AB, and by FCT-PestOE/SAU/UI4013 to iMed.ULisboa.

Ethical Statement The use of human samples was approved by the local institutional review board (IRB), both in Life and Health Sciences Research Institute (ICVS), Portugal, and VU University Medical Center Amsterdam, the Netherlands. Animal use complied with the Portuguese Law and the European Community Directive and followed the Federation of European Laboratory Animal Science Associations (FELASA) guidelines and recommendations concerning laboratorial animal welfare, being performed under the guidance of Adelaide Fernandes, with a FELASA level C certification (scientist), approved by the Portuguese DireçãoGeral de Veterinária. This ensured that any suffering or other harmful effects experienced by the animals were minimized and have been weighted against the potential benefits to humans.

\section{References}

1. Lassmann H, Bruck W, Lucchinetti CF (2007) The immunopathology of multiple sclerosis: an overview. Brain Pathol 17(2):210-218. doi:10.1111/j.1750-3639.2007.00064.x

2. Gerlach R, Demel G, Konig HG, Gross U, Prehn JH, Raabe A, Seifert V, Kogel D (2006) Active secretion of S100B from astrocytes during metabolic stress. Neuroscience 141(4):1697-1701. doi:10.1016/j.neuroscience.2006.05.008

3. Hachem S, Aguirre A, Vives V, Marks A, Gallo V, Legraverend C (2005) Spatial and temporal expression of S100B in cells of oligodendrocyte lineage. Glia 51(2):81-97. doi:10.1002/glia.20184

4. Donato R, Sorci G, Riuzzi F, Arcuri C, Bianchi R, Brozzi F, Tubaro C, Giambanco I (2009) S100B's double life: intracellular regulator and extracellular signal. Biochim Biophys Acta 1793(6):10081022. doi:10.1016/j.bbamcr.2008.11.009

5. Goncalves DS, Lenz G, Karl J, Goncalves CA, Rodnight R (2000) Extracellular S100B protein modulates ERK in astrocyte cultures. Neuroreport 11(4):807-809

6. Reali C, Scintu F, Pillai R, Donato R, Michetti F, Sogos V (2005) $\mathrm{S} 100 \mathrm{~b}$ counteracts effects of the neurotoxicant trimethyltin on astrocytes and microglia. J Neurosci Res 81(5):677-686. doi:10. 1002/jnr.20584

7. Selinfreund RH, Barger SW, Pledger WJ, Van Eldik LJ (1991) Neurotrophic protein S100 beta stimulates glial cell proliferation. Proc Natl Acad Sci U S A 88(9):3554-3558

8. Zhang L, Liu W, Alizadeh D, Zhao D, Farrukh O, Lin J, Badie SA, Badie B (2011) S100B attenuates microglia activation in gliomas: possible role of STAT3 pathway. Glia 59(3):486-498. doi:10.1002/ glia.21118

9. Ondruschka B, Pohlers D, Sommer G, Schober K, Teupser D, Franke H, Dressler J (2013) S100B and NSE as useful postmortem biochemical markers of traumatic brain injury in autopsy cases. J Neurotrauma 30(22):1862-1871. doi:10.1089/neu.2013.2895

10. Park JW, Suh GI, Shin HE (2013) Association between cerebrospinal fluid S100B protein and neuronal damage in patients with central nervous system infections. Yonsei Med J 54(3):567-571. doi: 10.3349 /ymj.2013.54.3.567

11. Huttunen HJ, Kuja-Panula J, Sorci G, Agneletti AL, Donato R, Rauvala H (2000) Coregulation of neurite outgrowth and cell survival by amphoterin and S100 proteins through receptor for advanced glycation end products (RAGE) activation. J Biol Chem 275(51):40096-40105. doi:10.1074/jbc.M006993200

12. Sorci G, Bianchi R, Riuzzi F, Tubaro C, Arcuri C, Giambanco I, Donato R (2010) S100B protein, a damage-associated molecular pattern protein in the brain and heart, and beyond. Cardiovasc Psychiatry Neurol. doi:10.1155/2010/656481

13. Astrand R, Unden J, Romner B (2013) Clinical use of the calciumbinding S100B protein. Methods Mol Biol 963:373-384. doi:10. 1007/978-1-62703-230-8 23

14. Ostendorp T, Leclerc E, Galichet A, Koch M, Demling N, Weigle B, Heizmann CW, Kroneck PM et al (2007) Structural and functional insights into RAGE activation by multimeric S100B. Embo J 26(16):3868-3878. doi:10.1038/sj.emboj.7601805

15. Michetti F, Massaro A, Murazio M (1979) The nervous systemspecific S-100 antigen in cerebrospinal fluid of multiple sclerosis patients. Neurosci Lett 11(2):171-175

16. Petzold A, Eikelenboom MJ, Gveric D, Keir G, Chapman M, Lazeron RH, Cuzner ML, Polman CH et al (2002) Markers for different glial cell responses in multiple sclerosis: clinical and pathological correlations. Brain 125(Pt 7):1462-1473

17. Rejdak K, Petzold A, Stelmasiak Z, Giovannoni G (2008) Cerebrospinal fluid brain specific proteins in relation to nitric oxide metabolites during relapse of multiple sclerosis. Mult Scler 14(1): 59-66. doi:10.1177/1352458507082061

18. Bartosik-Psujek H, Psujek M, Jaworski J, Stelmasiak Z (2011) Total tau and S100b proteins in different types of multiple sclerosis and during immunosuppressive treatment with mitoxantrone. Acta Neurol Scand 123(4):252-256. doi:10.1111/j.1600-0404.2010.01393

19. O'Connell KE, Mok T, Sweeney B, Ryan AM, Dev KK (2014) The use of cytokine signature patterns: separating drug naive, interferon and natalizumab-treated multiple sclerosis patients. Autoimmunity 47(8):505-511. doi:10.3109/08916934.2014.930734

20. Falcao AS, Silva RF, Vaz AR, Gomes C, Fernandes A, Barateiro A, Tiribelli C, Brites D (2013) Cross-talk between neurons and astrocytes in response to bilirubin: adverse secondary impacts. Neurotox Res. doi:10.1007/s12640-013-9427-y

21. van der Valk P, De Groot CJ (2000) Staging of multiple sclerosis (MS) lesions: pathology of the time frame of MS. Neuropathol Appl Neurobiol 26(1):2-10 
22. Kooi EJ, Prins M, Bajic N, Belien JA, Gerritsen WH, van Horssen J, Aronica E, van Dam AM et al (2011) Cholinergic imbalance in the multiple sclerosis hippocampus. Acta Neuropathol 122(3):313322. doi:10.1007/s00401-011-0849-4

23. Witte ME, Bo L, Rodenburg RJ, Belien JA, Musters R, Hazes T, Wintjes LT, Smeitink JA et al (2009) Enhanced number and activity of mitochondria in multiple sclerosis lesions. J Pathol 219(2):193204. doi:10.1002/path.2582

24. Sheridan GK, Dev KK (2012) S1P1 receptor subtype inhibits demyelination and regulates chemokine release in cerebellar slice cultures. Glia 60(3):382-392. doi:10.1002/glia.22272

25. Miron VE, Ludwin SK, Darlington PJ, Jarjour AA, Soliven B, Kennedy TE, Antel JP (2010) Fingolimod (FTY720) enhances remyelination following demyelination of organotypic cerebellar slices. Am J Pathol 176(6):2682-2694. doi:10.2353/ajpath.2010. 091234

26. Dyer JK, Bourque JA, Steeves JD (2005) The role of complement in immunological demyelination of the mammalian spinal cord. Spinal Cord 43(7):417-425. doi:10.1038/sj.sc.3101737

27. Sofroniew MV, Vinters HV (2010) Astrocytes: biology and pathology. Acta Neuropathol 119(1):7-35. doi:10.1007/s00401-009-0619-8

28. Inoue M, Shinohara ML (2013) NLRP3 Inflammasome and MS/ EAE. Autoimm Dis 2013:859145. doi:10.1155/2013/859145

29. Lu B, Wang H, Andersson U, Tracey KJ (2013) Regulation of HMGB1 release by inflammasomes. Protein Cell 4(3):163-167. doi:10.1007/s13238-012-2118-2

30. Rejdak K, Petzold A, Kocki T, Kurzepa J, Grieb P, Turski WA, Stelmasiak Z (2007) Astrocytic activation in relation to inflammatory markers during clinical exacerbation of relapsing-remitting multiple sclerosis. J Neural Transm 114(8):1011-1015. doi:10. 1007/s00702-007-0667-y

31. Missler U, Wiesmann M, Friedrich C, Kaps M (1997) S-100 protein and neuron-specific enolase concentrations in blood as indicators of infarction volume and prognosis in acute ischemic stroke. Stroke J Cerebr Circ 28(10):1956-1960

32. Wunderlich MT, Ebert AD, Kratz T, Goertler M, Jost S, Herrmann M (1999) Early neurobehavioral outcome after stroke is related to release of neurobiochemical markers of brain damage. Stroke J Cerebr Circ 30(6):1190-1195

33. Steinhoff BJ, Tumani H, Otto M, Mursch K, Wiltfang J, Herrendorf G, Bittermann HJ, Felgenhauer K et al (1999) Cisternal S100 protein and neuron-specific enolase are elevated and site-specific markers in intractable temporal lobe epilepsy. Epilepsy Res 36(1): 75-82

34. Herrmann M, Jost S, Kutz S, Ebert AD, Kratz T, Wunderlich MT, Synowitz H (2000) Temporal profile of release of neurobiochemical markers of brain damage after traumatic brain injury is associated with intracranial pathology as demonstrated in cranial computerized tomography. J Neurotrauma 17(2):113-122

35. Adami C, Sorci G, Blasi E, Agneletti AL, Bistoni F, Donato R (2001) S100B expression in and effects on microglia. Glia 33(2): 131-142

36. Donato R (2001) S100: a multigenic family of calcium-modulated proteins of the EF-hand type with intracellular and extracellular functional roles. Int J Biochem Cell Biol 33(7):637-668

37. Hidaka H, Endo T, Kawamoto S, Yamada E, Umekawa H, Tanabe K, Hara K (1983) Purification and characterization of adipose tissue S-100b protein. J Biol Chem 258(4):2705-2709

38. Takahashi K, Isobe T, Ohtsuki Y, Akagi T, Sonobe H, Okuyama T (1984) Immunohistochemical study on the distribution of alpha and beta subunits of S-100 protein in human neoplasm and normal tissues. Virchows Archiv B, Cell Pathol Includ Mol Pathol 45(4): 385-396

39. Koh SX, Lee JK (2014) S100B as a marker for brain damage and blood-brain barrier disruption following exercise. Sports Med 44(3):369-385. doi:10.1007/s40279-013-0119-9
40. Ortiz GG, Pacheco-Moises FP, Macias-Islas MA, Flores-Alvarado LJ, Mireles-Ramirez MA, Gonzalez-Renovato ED, HernandezNavarro VE, Sanchez-Lopez AL et al (2014) Role of the bloodbrain barrier in multiple sclerosis. Arch Med Res. doi:10.1016/j. arcmed.2014.11.013

41. Van Eldik LJ, Zimmer DB (1987) Secretion of S-100 from rat C6 glioma cells. Brain Res 436(2):367-370

42. Neeper M, Schmidt AM, Brett J, Yan SD, Wang F, Pan YC, Elliston K, Stern D et al (1992) Cloning and expression of a cell surface receptor for advanced glycosylation end products of proteins. J Biol Chem 267(21):14998-15004

43. Xie J, Reverdatto S, Frolov A, Hoffmann R, Burz DS, Shekhtman A (2008) Structural basis for pattern recognition by the receptor for advanced glycation end products (RAGE). J Biol Chem 283(40): 27255-27269. doi:10.1074/jbc.M801622200

44. Chan FK (2007) Three is better than one: pre-ligand receptor assembly in the regulation of TNF receptor signaling. Cytokine 37(2): 101-107. doi:10.1016/j.cyto.2007.03.005

45. Villarreal A, Aviles Reyes RX, Angelo MF, Reines AG, Ramos AJ (2011) S100B alters neuronal survival and dendrite extension via RAGE-mediated NF-kappaB signaling. J Neurochem 117(2):321332. doi:10.1111/j.1471-4159.2011.07207.x

46. Brozzi F, Arcuri C, Giambanco I, Donato R (2009) S100B protein regulates astrocyte shape and migration via interaction with $\mathrm{Src}$ kinase: implications for astrocyte development, activation, and tumor growth. J Biol Chem 284(13):8797-8811. doi:10.1074/jbc. M805897200

47. Bianchi R, Kastrisianaki E, Giambanco I, Donato R (2011) S100B protein stimulates microglia migration via RAGE-dependent upregulation of chemokine expression and release. J Biol Chem. doi:10. 1074/jbc.M110.169342

48. Bianchi R, Giambanco I, Donato R (2010) S100B/RAGEdependent activation of microglia via NF-kappaB and AP-1 Coregulation of COX-2 expression by $\mathrm{S} 100 \mathrm{~B}$, IL-1beta and TNFalpha. Neurobiol Aging 31(4):665-677. doi:10.1016/j. neurobiolaging.2008.05.017

49. Steiner J, Bernstein HG, Bogerts B, Gos T, Richter-Landsberg C, Wunderlich MT, Keilhoff G (2008) S100B is expressed in, and released from, OLN-93 oligodendrocytes: Influence of serum and glucose deprivation. Neuroscience 154(2):496-503. doi:10.1016/j. neuroscience.2008.03.060

50. Su Z, Yuan Y, Chen J, Zhu Y, Qiu Y, Zhu F, Huang A, He C (2011) Reactive astrocytes inhibit the survival and differentiation of oligodendrocyte precursor cells by secreted TNF-alpha. J Neurotrauma 28(6):1089-1100. doi:10.1089/neu.2010.1597

51. Deloulme JC, Raponi E, Gentil BJ, Bertacchi N, Marks A, Labourdette G, Baudier J (2004) Nuclear expression of S100B in oligodendrocyte progenitor cells correlates with differentiation toward the oligodendroglial lineage and modulates oligodendrocytes maturation. Mol Cell Neurosci 27(4):453-465. doi:10.1016/j.mcn. 2004.07.008

52. Compston A, Coles A (2008) Multiple sclerosis. Lancet 372(9648): 1502-1517. doi:10.1016/S0140-6736(08)61620-7

53. Villarreal A, Seoane R, Gonzalez Torres A, Rosciszewski G, Angelo MF, Rossi A, Barker PA, Ramos AJ (2014) S100B protein activates a RAGE-dependent autocrine loop in astrocytes: implications for its role in the propagation of reactive gliosis. J Neurochem. doi: $10.1111 /$ jnc. 12790

54. Cammer W, Zhang H (1999) Maturation of oligodendrocytes is more sensitive to TNF alpha than is survival of precursors and immature oligodendrocytes. J Neuroimmunol 97(1-2):37-42

55. Blakemore WF, Gilson JM, Crang AJ (2003) The presence of astrocytes in areas of demyelination influences remyelination following transplantation of oligodendrocyte progenitors. Exp Neurol 184(2):955-963. doi:10.1016/S0014-4886(03)00347-9 
56. Birgbauer E, Rao TS, Webb M (2004) Lysolecithin induces demyelination in vitro in a cerebellar slice culture system. J Neurosci Res 78(2):157-166. doi:10.1002/jnr.20248

57. Kotter MR, Setzu A, Sim FJ, Van Rooijen N, Franklin RJ (2001) Macrophage depletion impairs oligodendrocyte remyelination following lysolecithin-induced demyelination. Glia 35(3):204-212

58. Napoli I, Neumann H (2010) Protective effects of microglia in multiple sclerosis. Exp Neurol 225(1):24-28. doi:10.1016/j. expneurol.2009.04.024

59. Miron VE, Boyd A, Zhao JW, Yuen TJ, Ruckh JM, Shadrach JL, van Wijngaarden P, Wagers AJ et al (2013) M2 microglia and macrophages drive oligodendrocyte differentiation during CNS remyelination. Nat Neurosci 16(9):1211-1218. doi:10.1038/nn.3469

60. Rossi S, Studer V, Motta C, Germani G, Macchiarulo G, Buttari F, Mancino R, Castelli M et al (2014) Cerebrospinal fluid detection of interleukin-1 beta in phase of remission predicts disease progression in multiple sclerosis. J Neuroinflammation 11:32. doi:10.1186/ 1742-2094-11-32

61. Andersson A, Covacu R, Sunnemark D, Danilov AI, Dal Bianco A, Khademi M, Wallstrom E, Lobell A et al (2008) Pivotal advance: HMGB1 expression in active lesions of human and experimental multiple sclerosis. J Leukoc Biol 84(5):1248-1255. doi:10.1189/ jlb.1207844

62. Chen YC, Chen SD, Miao L, Liu ZG, Li W, Zhao ZX, Sun XJ, Jiang GX et al (2012) Serum levels of interleukin (IL)-18, IL-23 and IL-17 in Chinese patients with multiple sclerosis. J Neuroimmunol 243(1-2):56-60. doi:10.1016/j.jneuroim.2011.12.008

63. Jha S, Srivastava SY, Brickey WJ, Iocca H, Toews A, Morrison JP, Chen VS, Gris D et al (2010) The inflammasome sensor, NLRP3, regulates CNS inflammation and demyelination via caspase-1 and interleukin-18. J Neurosci 30(47):15811-15820. doi:10.1523/ JNEUROSCI.4088-10.2010

64. Robinson AP, Caldis MW, Harp CT, Goings GE, Miller SD (2013) High-mobility group box 1 protein (HMGB1) neutralization ameliorates experimental autoimmune encephalomyelitis. J Autoimmun 43:32-43. doi:10.1016/j.jaut.2013.02.005

65. Gris D, Ye Z, Iocca HA, Wen H, Craven RR, Gris P, Huang M, Schneider M et al (2010) NLRP3 plays a critical role in the development of experimental autoimmune encephalomyelitis by mediating Th1 and Th17 responses. J Immunol 185(2):974-981. doi:10. 4049/jimmunol.0904145

66. Inoue M, Williams KL, Gunn MD, Shinohara ML (2012) NLRP3 inflammasome induces chemotactic immune cell migration to the CNS in experimental autoimmune encephalomyelitis. Proc Natl Acad Sci U S A 109(26):10480-10485. doi:10.1073/pnas. 1201836109

67. Yan SS, Wu ZY, Zhang HP, Furtado G, Chen X, Yan SF, Schmidt AM, Brown C et al (2003) Suppression of experimental autoimmune encephalomyelitis by selective blockade of encephalitogenic T-cell infiltration of the central nervous system. Nat Med 9(3):287293. doi: $10.1038 / \mathrm{nm} 831$

68. Turco F, Sarnelli G, Cirillo C, Palumbo I, De Giorgi F, D'Alessandro A, Cammarota M, Giuliano M et al (2014) Enteroglial-derived S100B protein integrates bacteria-induced Toll-like receptor signalling in human enteric glial cells. Gut 63(1):105-115. doi:10.1136/gutjnl-2012-302090

69. Deane R, Singh I, Sagare AP, Bell RD, Ross NT, LaRue B, Love R, Perry $S$ et al (2012) A multimodal RAGE-specific inhibitor reduces amyloid beta-mediated brain disorder in a mouse model of Alzheimer disease. J Clin Invest 122(4):1377-1392. doi:10.1172/ JCI58642

70. Pan W, Xin P, Patrick S, Dean S, Keating C, Clawson G (2010) Primer-free aptamer selection using a random DNA library. J Vis Exp (41). doi:10.3791/2039

71. Farooq SM, Elkhatib WF, Ashour HM (2014) The in vivo and in vitro induction of anterior chamber associated immune deviation to myelin antigens in C57BL/6 mice. Brain Behav Immun 42:118122. doi:10.1016/j.bbi.2014.06.010 